Review

\title{
Cutting-Edge High-Power Ultrafast Thin Disk Oscillators
}

Clara J. Saraceno ${ }^{1{ }^{*},}$, Cinia Schriber ${ }^{1}$, Florian Emaury ${ }^{1}$, Oliver H. Heckl ${ }^{1}$, Cyrill R. E. Baer ${ }^{1}$, Martin Hoffmann ${ }^{1}$, Kolja Beil ${ }^{2}$, Christian Kränkel ${ }^{2,3}$, Matthias Golling ${ }^{1}$, Thomas Südmeyer ${ }^{1,4}$ and Ursula Keller ${ }^{1}$

1 Department of Physics, Institute for Quantum Electronics, ETH Zurich, Zurich 8093, Switzerland; E-Mails: cschriber@phys.ethz.ch (C.S.); emaury@phys.ethz.ch (F.E.); heckl@phys.ethz.ch (O.H.H.); cbaer@phys.ethz.ch (C.R.E.B.); mh@phys.ethz.ch (M.H.); golling@phys.ethz.ch (M.G.); thomas.sudmeyer@unine.ch (T.S.); keller@phys.ethz.ch (U.K.)

2 Institute of Laser-Physics, University of Hamburg, Luruper Chaussee 149, Hamburg 22761, Germany; E-Mails: kbeil@physnet.uni-hamburg.de (K.B.), kraenkel@physnet.uni-hamburg.de (C.K.)

3 The Hamburg Centre for Ultrafast Imaging, Luruper Chaussee 149, Hamburg 22761, Germany

4 Time and Frequency Laboratory, Department of Physics, University of Neuchâtel, Neuchâtel 2000, Switzerland

* Author to whom correspondence should be addressed; E-Mail: saraceno@phys.ethz.ch; Tel.: +41-44-633-3288; Fax: +41-44-633-1059.

Received: 21 January 2013; in revised form: 22 February 2013 / Accepted: 25 February 2013 / Published: 2 April 2013

Abstract: A growing number of applications in science and industry are currently pushing the development of ultrafast laser technologies that enable high average powers. SESAM modelocked thin disk lasers (TDLs) currently achieve higher pulse energies and average powers than any other ultrafast oscillator technology, making them excellent candidates in this goal. Recently, $275 \mathrm{~W}$ of average power with a pulse duration of 583 fs were demonstrated, which represents the highest average power so far demonstrated from an ultrafast oscillator. In terms of pulse energy, TDLs reach more than $40 \mu \mathrm{J}$ pulses directly from the oscillator. In addition, another major milestone was recently achieved, with the demonstration of a TDL with nearly bandwidth-limited 96-fs long pulses. The progress achieved in terms of pulse duration of such sources enabled the first measurement of the carrier-envelope offset frequency of a modelocked TDL, which is the first key step towards full stabilization of such a source. We will present the key elements that enabled these latest results, as well as an outlook towards the next scaling steps in average power, pulse 
energy and pulse duration of such sources. These cutting-edge sources will enable exciting new applications, and open the door to further extending the current performance milestones.

Keywords: ultrafast laser; high-power laser; semiconductor saturable absorber mirror (SESAM); thin disk laser

\section{Introduction}

Ultrafast lasers sources are one of the main scientific achievements of the past decades. In addition to an important number of industrial applications, such as high-speed and high-precision micromachining they have tremendous impact on many disciplines of scientific research for example in biology, chemistry, physics, materials science and medicine, where they have become essential tools [1]. These sources generate intense and very short laser light bursts (with durations of femtoseconds $=10^{-15} \mathrm{~s}$ to picoseconds $=10^{-12} \mathrm{~s}$ ) and can be tightly focused in space, reaching electric field strengths comparable to those binding electrons to atoms and molecules. This enables the study of the interaction of matter with these strong fields and allows us to temporally resolve complex dynamic processes that occur on this timescale. This research topic is most widely referred to as strong-field physics.

Nowadays, most laboratory-based ultrafast laser systems for such applications rely on complex amplifier systems based on Ti:sapphire technology that can deliver ultrashort pulses $(<30 \mathrm{fs})$ with GWs of peak power, sufficient to reach the necessary electric field strength to carry out the targeted experiments. However, in addition to being complex and expensive, these sources are limited to repetition rates in the kilohertz range, with only a few watts of average power.

The development of novel ultrafast sources that operate at higher average power is currently a topic of important research efforts. The combination of high peak power and high average power is very attractive for the above-mentioned strong-field applications. Driving such experiments at multimegahertz repetition rate results in a reduced measurement time and a higher signal-to-noise ratio. Furthermore, photoionization studies in noble gases show that space charge issues are also reduced at higher repetition rates [2]. An important example where such sources would have major impact is high harmonic generation (HHG) [3,4] where driving the experiments with high-average power sources opens new avenues for increasing the vacuum ultraviolet and extreme ultraviolet (VUV/XUV) photon flux [5], possibly in combination with enhanced phase-matching techniques (such as hollow-core photonic crystal fibers (HC-PCF) [6], or resonant field enhancement in nanostructured targets [7]). Such compact megahertz sources of radiation in the VUV/XUV spectral region would enable coherent sources to be available at a wavelength range where laser transitions are not known to date.

The main technological challenges that arise from the combination of high average and peak powers are mainly an excessive heat deposition in the gain medium and a too large nonlinearity accumulated by the pulses during propagation. In the past few years, several clever amplifier geometries have been suggested to overcome these limitations such as slab amplifiers [8], fiber based chirped pulse amplifiers (CPA) [9], and thin-disk laser (TDL) amplifiers [10]. However, these amplifier technologies require a low-power seed oscillator and several amplifying stages to reach the targeted high average power, resulting in overall complex systems. 
In contrast, semiconductor saturable absorber mirror (SESAM) modelocked TDLs enable high average powers and femtosecond operation directly from a single oscillator, without the need for additional amplification stages. Unlike bulk oscillators, where thermal aberrations that occur in the gain medium limit the achievable output power, the TDL concept $[11,12]$ is based on a very thin disk-shaped gain medium that can be efficiently cooled through the backside. The resulting outstanding heat removal capabilities allow for high average powers and excellent beam quality suitable for SESAM modelocking. In addition, the very thin gain medium is ideally suited for small amounts of accumulated nonlinearity even at very high peak powers.

Furthermore, modelocking using semiconductor saturable absorber mirrors (SESAMs) is currently the best-suited approach for high-power ultrafast laser oscillators. The invention of the SESAM nearly 20 years ago [13-15] represented an important breakthrough in the development of more practical and robust ultrafast laser sources. Today, SESAMs have become key devices for modelocking numerous laser types, including diode-pumped solid-state lasers, fiber lasers, and semiconductor lasers. Semiconductors are ideally suited for saturable absorbers because they can cover a broad wavelength range and yield short recovery times, supporting the generation of ultrashort pulse durations. The macroscopic parameters for modelocking can be optimized over a wide range by the design and growth conditions of the mirror structure and the choice of the semiconductor absorber.

The combination of the SESAM and the TDL concepts results in a power scalable ultrafast technology. Simply increasing the pump power with both an increased pump spot area on the disk and laser spot area on the SESAM allows one to increase the output power of SESAM modelocked TDLs. This resulted in a steady increase of the average power and pulse energy available from such modelocked sources since their first demonstration in the year 2000 [16] (Figure 1). Currently, SESAM modelocked TDLs achieve higher average powers $(>275 \mathrm{~W}[17])$ and pulse energies $(>40 \mu \mathrm{J}[18])$ than any other oscillator technology.

Figure 1. Evolution of pulse energy (a) and average power (b) of ultrafast semiconductor saturable absorber mirrors (SESAM)-modelocked thin-disk laser (TDLs). Most power and energy scaling was achieved using the well-established gain material Yb:YAG, but promising new materials for this application are currently the topic of important research efforts.
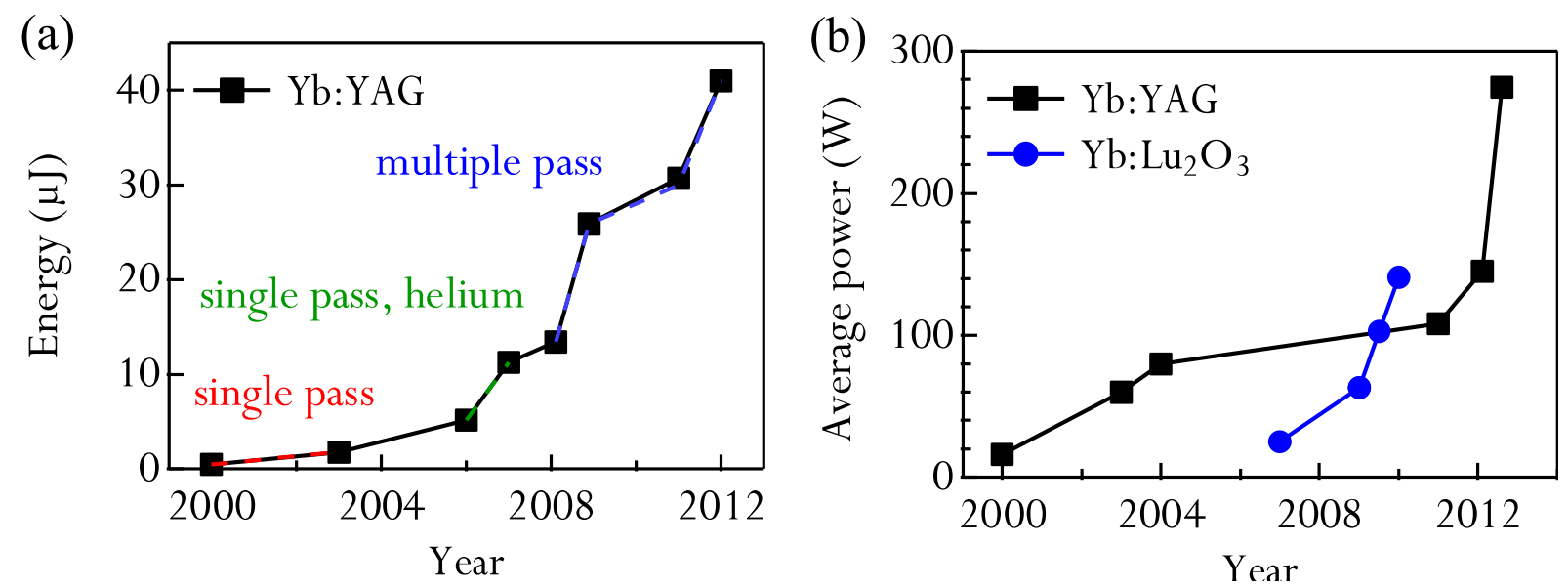

In this paper, we will review recent progress in the performance of modelocked TDLs. The average power of ultrafast oscillators recently reached a new limit with the demonstration of a SESAM 
modelocked TDL with $275 \mathrm{~W}$ - the highest average power reported from an ultrafast oscillator to date. The laser was based on the gain material $\mathrm{Yb}: \mathrm{YAG}$ and operated with a pulse duration of $583 \mathrm{fs}$ and a pulse energy of $16.9 \mu \mathrm{J}$. This performance was obtained by operating the modelocked laser in a vacuum environment to eliminate the parasitic nonlinearity of the air inside the oscillator. We will summarize the results obtained using this new approach, which represents an important step forward towards the kilowatt average power level milestone. Another key element to achieve this latest step in power scaling was the availability of SESAMs with high-damage threshold and parameters suitable for high-power operation. A recent investigation, which targeted to specifically explore damage behavior of SESAMs for such high-power oscillators [19], enabled the fabrication of such robust samples. We will review the guidelines developed in this recent study and discuss future improvements.

Another major milestone for modelocked TDLs is to extend the high-power capabilities of TDLs to the sub-100 fs regime. This topic is intimately linked to the development of novel broadband materials suitable for this geometry [20]. Recently, the limits in terms of pulse duration of modelocked TDLs based on different gain materials were explored. As a result, a first important step in this direction was achieved, with the demonstration of sub-100 fs pulses from a TDL based on the sesquioxide gain material $\mathrm{Yb}: \mathrm{LuScO}_{3}(\mathrm{Yb}: \mathrm{LuScO})$ [21]. In this first experiment, sub-100 fs operation was achieved at moderate average output powers $(5 \mathrm{~W})$. However, preliminary power scaling experiments indicate that much higher output powers are within reach. Furthermore, the intracavity peak power levels achieved are already high enough for preliminary intralaser nonlinear optics experiments, which is a promising application [22].

The progress achieved in the pulse duration of TDLs enabled us to explore for the first time the carrier-envelope phase properties of a TDL based on $\mathrm{Yb}: \mathrm{Lu}_{2} \mathrm{O}_{3}(\mathrm{Yb}: \mathrm{LuO})$ that delivered $7 \mathrm{~W}$ and $142 \mathrm{fs}$ pulses. We measured the carrier-envelope offset (CEO) frequency, which is the first key step towards full stabilization of such a source [22]. This experiment shows that TDLs are also excellent candidates for applications in spectroscopy and metrology, where high-power frequency combs are of interest.

Finally, we will conclude with an outlook towards higher average powers, higher pulse energies and higher peak powers from TDLs.

\section{Average Power Scaling of Modelocked Thin-Disk Lasers: Challenges and Milestones}

Several issues have, so far, limited scaling of modelocked TDLs to average output powers in the kilowatt range. One challenge is achieving fundamental transverse mode operation at high average powers, which is a crucial aspect for stable passive modelocking. As a result, average power and energy scaling of passively modelocked TDLs goes hand-in-hand with progress in power scaling of TDLs operating in single fundamental transverse mode. In most bulk lasers, thermal aberrations that occur in the gain medium mainly limit the available power. TDLs are ideally suited to partially overcome this limitation. Significant efforts have been carried out in the past years to optimize fabrication and contacting of standard thin gain media to minimize these thermal aberrations, in particular for the most commonly used gain material for TDLs, Yb:YAG [12,23,24]. Until now, $500 \mathrm{~W}$ of continuous wave (cw) power with diffraction limited beam quality $\left(M^{2}<1.1\right)$ have been demonstrated using one disk based on this material [25]. For novel gain materials such as $\mathrm{Yb}: \mathrm{LuO}$ or $\mathrm{Yb}: \mathrm{LuScO}$, small residual thermal lensing can be compensated by adapting the resonator design [26]. These novel 
materials such as $\mathrm{Yb}: \mathrm{LuO}$ have a large potential to outperform Yb:YAG in terms of efficiency and pulse duration, when growth and contacting techniques are perfected. The challenges of achieving single-transverse mode operation from high-power TDLs in the context of modelocking have already been discussed in detail in reference [27]. Here, we will focus on the challenges of achieving soliton modelocking at high average powers and high pulse energies.

One important point is avoiding an excessive nonlinear phase shift due to self-phase modulation (SPM) that can destabilize the pulses in the soliton modelocking regime [28,29]. In the past, the dominant source of unwanted SPM was the nonlinearity of air. We will discuss different approaches that have been demonstrated in the past years to reduce this parasitic nonlinearity. In particular, a new approach to overcome this limitation in the context of modelocked TDLs was recently demonstrated, which consists of operating the oscillator in a vacuum environment. In this way, the nonlinearity of the ambient environment is reduced by several orders of magnitude enabling high average powers and pulse energies in simple oscillator geometries, with a low number of passes through the gain medium and low output coupling rates. Furthermore, only a small amount of dispersion is required even at very high intracavity pulse energy to compensate for this phase shift within one round-trip in the laser cavity. With this approach, the latest step in average power scaling was achieved, reaching an average power of $275 \mathrm{~W}$ at a pulse duration of $583 \mathrm{fs}$ using the well-established gain material Yb:YAG. The laser operates at a repetition rate of $16.3 \mathrm{MHz}$ resulting in a pulse energy of $16.9 \mu \mathrm{J}$ and a peak power of 25.6 MW. We will review this latest step in power scaling, which opens the door to future kilowattlevel oscillators.

Another point that has only recently been investigated is possible limitations of SESAMs in terms of damage and lifetime. As a result of a detailed investigation, simple guidelines to design robust SESAMs with high-damage thresholds and optimized parameters for operation at extreme intracavity conditions were recently developed [19]. This is a crucial point for future kilowatt-level oscillators. We will review these guidelines and discuss further improvements.

Ultimately, thermal effects and damage that occur in different cavity components limit average power scaling of modelocked TDLs. This is particularly the case in simple thin disk oscillator geometries with low gain per roundtrip, where the circulating intracavity power can reach several kilowatts of average power. We will discuss critical points and future improvements.

\subsection{Harnessing Intracavity Nonlinearity}

In a typical cavity for a soliton modelocked TDL (Figure 2), the circulating pulse experiences SPM by propagating through nonlinear materials like the gain medium, a Brewster plate and the air atmosphere. Most commonly, the disk is used as a folding mirror in a linear cavity, resulting in 4 gain passes through the thin disk. This configuration is usually referred to as a "single-pass" configuration. A Brewster plate is most commonly used to obtain a linearly polarized output and for fine adjustment of the SPM by placing it at a position in the cavity where there is a focus. Negative dispersion is introduced with dispersive mirrors throughout the cavity. Most commonly, Gires Tournois Interferometer (GTI)-type mirrors are used because they can provide large amounts of negative dispersion [30,31] required for stable soliton modelocking at the pulse durations typically obtained in state-of-the-art TDLs. 
Figure 2. Typical layout of a modelocked TDL cavity. HR: highly-reflective mirror, OC: output coupling mirror, DM: dispersive mirror.

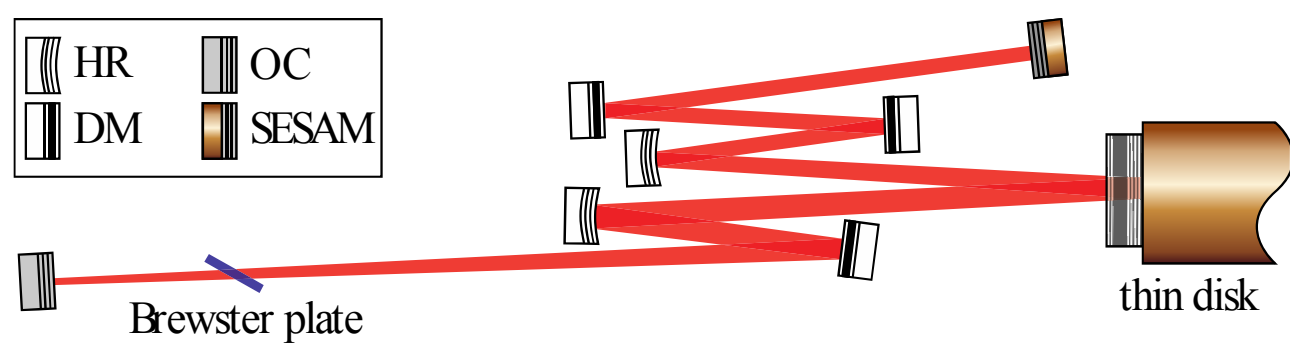

The total nonlinear phase shift $\Phi_{\mathrm{nl}}$ accumulated by the pulses due to self-phase modulation (SPM) per cavity round trip is given by

$$
\phi_{\mathrm{nl}}=2 \cdot \frac{2 \pi}{\lambda} \int_{\mathrm{cav}} n_{2}(z) I(z) d z
$$

Most commonly, the $\gamma$-factor (in $\operatorname{mrad} / \mathrm{MW}$ ) is used, which is independent of the pulse parameters but takes into account the different spot sizes of the beam throughout the cavity.

$$
\phi_{\mathrm{nl}}=4 \cdot \frac{2 \pi}{\lambda} P_{\mathrm{pk}} \int_{\mathrm{cav}} \frac{n_{2}(z)}{A(z)} d z=P_{\mathrm{pk}} \cdot \gamma_{\mathrm{cav}}
$$

Therefore, the $\gamma$-factor can be written as:

$$
\gamma_{\mathrm{cav}}=4 \cdot \frac{2 \pi}{\lambda} \int_{\mathrm{cav}} \frac{n_{2}(z)}{A(z)} d z
$$

Different elements in the cavity contribute to the total $\gamma$-factor and the total nonlinear phase shift undergone by the pulses. In Table 1, the contribution of these different elements to the total nonlinear phase shift in state-of-the-art modelocked thin disk lasers is presented.

Table 1. Summary of performance and contribution of different cavity elements to nonlinear phase shift due to self-phase modulation (SPM) in state-of-the-art high-power TDLs.

\begin{tabular}{lcccc}
\hline \multicolumn{1}{c}{ Parameter } & Yb:YAG [32] & Yb:YAG [17] & Yb:YAG [18] & Yb:LuO [26] \\
\hline Average output power & $44 \mathrm{~W}$ & $275 \mathrm{~W}$ & $145 \mathrm{~W}$ & $141 \mathrm{~W}$ \\
Repetition rate & $4 \mathrm{MHz}$ & $16.3 \mathrm{MHz}$ & $3.5 \mathrm{MHz}$ & $60 \mathrm{MHz}$ \\
Pulse duration & $791 \mathrm{fs}$ & $583 \mathrm{fs}$ & $1100 \mathrm{fs}$ & $740 \mathrm{fs}$ \\
Output pulse energy & $11.3 \mu \mathrm{J}$ & $16.9 \mu \mathrm{J}$ & $41 \mu \mathrm{J}$ & $2.35 \mu \mathrm{J}$ \\
Output peak power & $12.6 \mathrm{MW}$ & $25.6 \mathrm{MW}$ & $33 \mathrm{MW}$ & $2.8 \mathrm{MW}$ \\
Intracavity average power & $450 \mathrm{~W}$ & $2.5 \mathrm{~kW}$ & $0.2 \mathrm{~kW}$ & $1.5 \mathrm{~kW}$ \\
Intracavity pulse energy & $113 \mu \mathrm{J}$ & $154 \mu \mathrm{J}$ & $57 \mu \mathrm{J}$ & $25 \mu \mathrm{J}$ \\
Intracavity peak power & $127 \mathrm{MW}$ & $236 \mathrm{MW}$ & $46 \mathrm{MW}$ & $30 \mathrm{MW}$ \\
Nonlinear phase shift environment $\Phi_{\text {env }}$ & helium $108 \mathrm{mrad}$ & vacuum $8 \mathrm{mrad}$ & air $1725 \mathrm{mrad}$ & air $44 \mathrm{mrad}$ \\
Nonlinear phase shift & $95 \mathrm{mrad}$ & $17 \mathrm{mrad}$ & - & - \\
Brewster plate $\Phi_{\mathrm{BP}}$ & $19 \mathrm{mrad}$ & $3 \mathrm{mrad}$ & & - \\
Nonlinear phase shift disk $\Phi_{\text {disk }}$ & - & $45 \mathrm{mrad}$ & - & $4 \mathrm{mrad}$ \\
Other nonlinear phase shifts $\Phi_{\text {other }}$ & $222 \mathrm{mrad}$ & $75 \mathrm{mrad}$ & $1725 \mathrm{mrad}^{2}$ & $48 \mathrm{mrad}$ \\
Total nonlinear phase shift $\Phi_{\text {cav }}$ & $-20,000 \mathrm{fs}^{2}$ & $-8,100 \mathrm{fs}^{2}$ & $-346,500 \mathrm{fs}^{2}$ & $-9,900 \mathrm{fs}^{2}$ \\
GDD per roundtrip & & & & \\
\hline
\end{tabular}


- Gain material:

The SPM from the disk can typically be neglected as the laser mode size on the disk is large and the pulse passes only through very little material.

- Brewster plate:

The influence of the Brewster plate can be controlled by the choice of the thickness, the material and the mode size at the location in the cavity where it is placed. Usually, the presence of a Brewster plate is beneficial as it offers control over the total amount of SPM when placed at a position in the cavity close to a focus, and ensures linear polarization of the laser output. In cavities where a fine control of the SPM can be achieved otherwise (for example with the air pressure, when the oscillator is operated in a vacuum chamber) a thin-film polarizer can be chosen instead of a Brewster plate to select the polarization.

- Cavity optics:

The nonlinearity introduced by the coatings of different cavity optics has been, until now, always considered negligible. However, in simple cavities where a low number of passes through the gain medium is chosen, they can become an important contribution to the total soliton phase shift at extreme intracavity peak powers.

- Air atmosphere:

The contribution of the air atmosphere was initially ignored, since the nonlinear refractive index $n_{2, \text { atm }}$ is orders of magnitude smaller than the refractive index of, for example, a fused-silica Brewster plate. However, typical MHz TDLs have cavity lengths in the order of several meters to several tens of meters. Furthermore, in most modelocked TDLs, the intracavity peak power is substantially higher than the output peak power and can exceed $100 \mathrm{MW}$ [17,32]. Therefore, intracavity SPM introduced by the ambient air in the cavity can become the main contribution to the total soliton phase shift [33]. In order to compensate for this phase shift and obtain stable soliton modelocking, large amounts of negative GDD are required. If this phase shift becomes excessive (typically larger than some hundred mrad), modelocked operation is destabilized [28,34]. Different approaches have been suggested in the past years to overcome this limitation:

- Helium flooding

Helium has a nonlinear refractive index $n_{2}$ that is approximately 8 times smaller than air ( $n_{2, \text { air }} \approx 3 \cdot 10^{-23} \mathrm{~m}^{2} / \mathrm{W},[35]$ and $n_{2, \mathrm{He}} \approx 0.4 \cdot 10^{-23} \mathrm{~m}^{2} / \mathrm{W}[36]$ ). Therefore, replacing the air in the cavity by helium enabled the demonstration of the $10 \mu \mathrm{J}$ pulse energy milestone in 2008 . In this result, $11 \mu \mathrm{J}$ were obtained at an average power of $44 \mathrm{~W}$ from a SESAM modelocked TDL based on Yb:YAG [32]. In this oscillator, the disk was used in a single-pass configuration, resulting in a high intracavity pulse energy of $>110 \mu \mathrm{J}$. The required dispersion to generate the 791-fs pulses was, in this case, $-20,000 \mathrm{fs}^{2}$.

- Multiple gain passes through the same disk

Another approach to lower the SPM in the cavity is to reduce the intracavity peak power by using a higher output coupler transmission. An important advantage of this approach is that the fluence on the SESAM and the thermal load on all components inside the laser cavity are reduced. Efficient laser operation with a higher output coupling transmission is only possible if the gain per cavity round trip is increased accordingly. This can be achieved by multiplying 
the number of passes through the same gain disk, which results in an increased overall gain per cavity roundtrip. This geometry, most commonly referred to as "active multi-pass cell" in a modelocked TDL, was introduced by Neuhaus et al. [37,38]. Using this approach, $145 \mathrm{~W}$ of average power were demonstrated with a pulse energy of $41 \mu \mathrm{J}$ using a $72 \%$ output coupling transmission and 11 passes through the Yb:YAG thin disk [18]. In this case, the intracavity pulse energy was below $60 \mu \mathrm{J}$ and operation in air was possible with a total GDD of $-236,000 \mathrm{fs}^{2}$. In addition to the large amounts of negative GDD required in this approach, a large amount of passes through the thin disk significantly increases the demands on the disk quality, since the cavity stability zones shrink significantly with the number of passes [27]. Another potential disadvantage is the reduced $Q$-factor of the cavity, resulting in a higher intrinsic noise level. Furthermore, a larger gain per roundtrip results in longer minimum achievable pulse duration [28,29]. This is not an issue in industrial applications such as highspeed micromachining, where obtaining short pulse duration is not of critical importance. In the case of scientific applications, shorter pulse durations are more critical, in particular since it simplifies further pulse compression schemes.

- $\quad$ Multiple gain passes through different disks

The combination of several laser heads in one cavity has already been demonstrated [12], but not in the context of modelocked TDLs. In this case the main difficulty is to achieve fundamental mode operation, as each disk can show a different thermal lensing behavior.

- Vacuum environment

Operating the oscillator in vacuum by placing it in a vacuum chamber allows for a reduction of the nonlinearity of the ambient environment by several orders of magnitude, since the nonlinear refractive index $n_{2}$ parameter varies linearly with air pressure for medium vacuum levels [39]. In addition to a minimal nonlinear phase shift at very high intracavity peak power levels, the advantages of operating the oscillator in vacuum are many-fold:

- Fine adjustment of the air pressure inside the cavity enables one to tune the nonlinear phase shift and minimize the pulse duration at a given output power. This eliminates the need for a moving Brewster plate in the cavity that can introduce aberrations and small losses.

- The low amount of nonlinearities allows one to operate in simple oscillator geometries with low gain per cavity round-trip (one or two passes on the disk used as a folding mirror in the cavity).

- Only small amounts on negative GDD are required to compensate for the small nonlinear phase shifts even at high intracavity peak powers. This results in lower parasitic losses and thermal effects that can occur in the dispersive mirrors.

- Turbulences of air and related pointing instabilities are minimized.

- Operating in a moderate vacuum environment allows for keeping the oscillator optics clean, which is critical at high intracavity powers.

- In addition to the above-mentioned points, developing robust vacuum oscillator technology will facilitate future intracavity HHG experiments, for which operation in 
vacuum is essential to avoid UV-light absorption by air. Furthermore, for other nonlinear experiments, one could consider flooding the chamber with other gases.

On the other hand, heat convection due to air is nearly completely eliminated by operating the oscillator in vacuum. In our experiment, we observed increased thermal effects in critical optics such as the dispersive mirrors when the oscillator was operated in vacuum. Nevertheless, using thermally improved mirrors and/or actively cooling these critical elements in the cavity (in the same way the disk or the SESAM are cooled) can solve these issues.

The advantages of this promising new approach were recently confirmed for the first time with the demonstration of a thin disk oscillator with $275 \mathrm{~W}$ [17]. In the next paragraph, we will summarize this recently achieved power scaling result which paves the way to even higher average output powers and pulse energies from such ultrafast oscillators.

\subsection{State-of-the Art Modelocked Thin-Disk Laser with $275 \mathrm{~W}$ of Average Power}

\subsubsection{Experimental Setup and Results}

The gain element used was a commercial Yb:YAG thin disk glued on a water-cooled diamond heatsink (TRUMPF GmbH). The disk was $\approx 100 \mu \mathrm{m}$ thick. The thin-disk head was arranged for 24 pump passes through the disk and a pump spot diameter of $4.7 \mathrm{~mm}$. The disk was pumped at its broad absorption line at $940 \mathrm{~nm}$. The measurement of the thermal lensing of this disk showed no significant thermal lensing over the whole pump power range used throughout the experiment, which allowed for robust fundamental transverse mode operation. We used an outcoupling rate of $11.4 \%$ for the modelocking experiment, and obtained up to $340 \mathrm{~W}$ of $\mathrm{cw}$ power at an optical-to-optical efficiency of $39.2 \%$ with a diffraction-limited beam $\left(M^{2}<1.05\right)$ (Figure 3$)$.

Figure 3. (a) cw fundamental transverse-mode operation with the same output coupler as for the modelocking experiment, using only highly reflective mirrors as cavity mirrors and without polarization control of the laser; (b) $M^{2}$ measurement at the maximum power of $340 \mathrm{~W}$. Inset: Picture of the laser mode at the maximum cw output power.

(a)

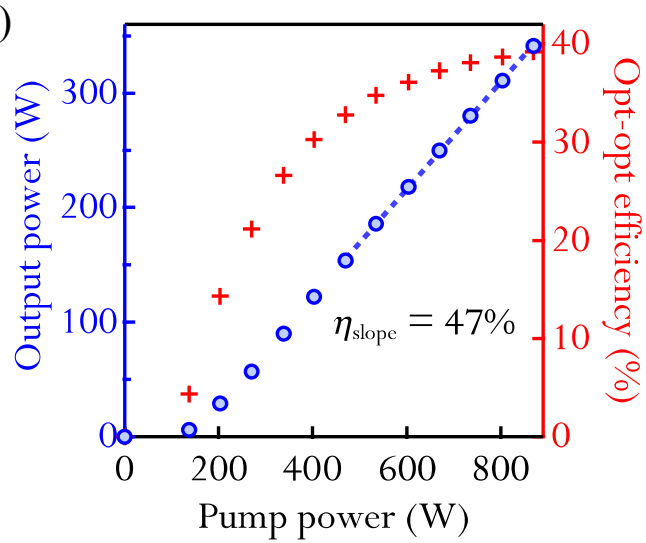

(b)

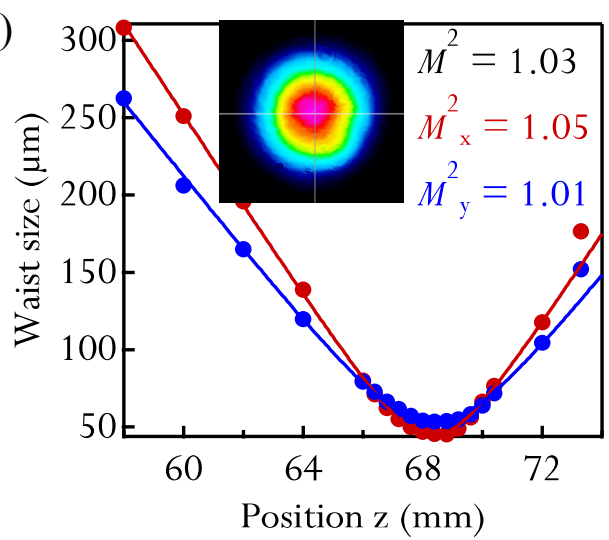

A set of five dispersive mirrors in the cavity introduced approximately $-8100 \mathrm{fs}^{2}$ of negative GDD per roundtrip, required for soliton modelocking. A fused silica plate with a thickness of $700 \mu \mathrm{m}$ was inserted at Brewster's angle for polarization selection. It was introduced at a fixed position in the 
cavity where the beam had a large radius of $\approx 1.3 \mathrm{~mm}$. This controls the laser polarization with minimal SPM. Fine control of the total SPM was achieved by changing the pressure in the vacuum chamber by introducing small amounts of nitrogen.

The SESAM used in this experiment was designed for high damage threshold and high-power modelocking following the guidelines presented in reference [19] and summarized in paragraph 2.3 of this paper. Special attention was paid during fabrication to obtain a large homogeneous sample for future spot size scaling (Figure 4). It consists of a distributed Bragg reflector (DBR) and three 10-nm InGaAs quantum wells (QWs) as absorbers in an antiresonant configuration. A dielectric topcoating that consists of 3 quarter-wave pairs of $\mathrm{SiO}_{2} / \mathrm{Si}_{3} \mathrm{~N}_{4}$ was deposited by plasma enhanced chemical vapor deposition (PECVD). Nonlinear reflectivity and recovery measurements of this sample yielded a saturation fluence $F_{\text {sat }}=140 \mu \mathrm{J} / \mathrm{cm}^{2}$, a modulation depth $\Delta R=0.95 \%$, nonsaturable losses $\Delta R_{\mathrm{ns}}=0.1 \%$ and a recovery time $\tau_{1 / \mathrm{e}}=67 \mathrm{ps}$. The vacuum chamber (Figure 4 ) was operated at a constant air pressure of 0.5 mbar, which was the lowest value that could be obtained in our setup and with the available vacuum pump.

Stable cw modelocking was obtained for average powers ranging from $135 \mathrm{~W}$ to $275 \mathrm{~W}$ (Figure $5 \mathrm{~d}$ ). When trying to reach higher power levels, the optical-to-optical efficiency decreased but no modelocking instabilities were observed. In order to avoid possible damage of the thin disk, the pump power was not further increased. At the maximum power of $275 \mathrm{~W}$, the pulse duration was $583 \mathrm{fs}$ (Figure 5a). The optical-to-optical efficiency was 32.4\%, corresponding to an incident pump power of $839 \mathrm{~W}$. The pulses had a time bandwidth product of 0.329 (ideal $\operatorname{sech}^{2} 0.315$ ), determined with the measured spectral bandwidth of $2 \mathrm{~nm}$ (Figure 5b). The repetition rate of the pulses was $16.3 \mathrm{MHz}$ (Figure 5c), resulting in a pulse energy of $16.9 \mu \mathrm{J}$. The corresponding peak power of the pulses is 25.6 MW. Operation with a single pulse circulating in the cavity was confirmed using a fast photodiode $(25 \mathrm{GHz})$ and a sampling oscilloscope. Furthermore, the delay of the autocorrelator ( $80 \mathrm{ps)}$ was scanned in search for cross-correlations of potential parasitic pulses with the main pulse. The beam at the maximum modelocked average power level was nearly diffraction limited with an $M^{2}<1.05$.

Figure 4. Vacuum chamber where the oscillator was built and schematic setup of the 16.3 $\mathrm{MHz}$ pulse repetition rate fundamental transverse mode cavity used for the high-power modelocking experiment. Inset: Picture of large scale SESAM used for this experiment.
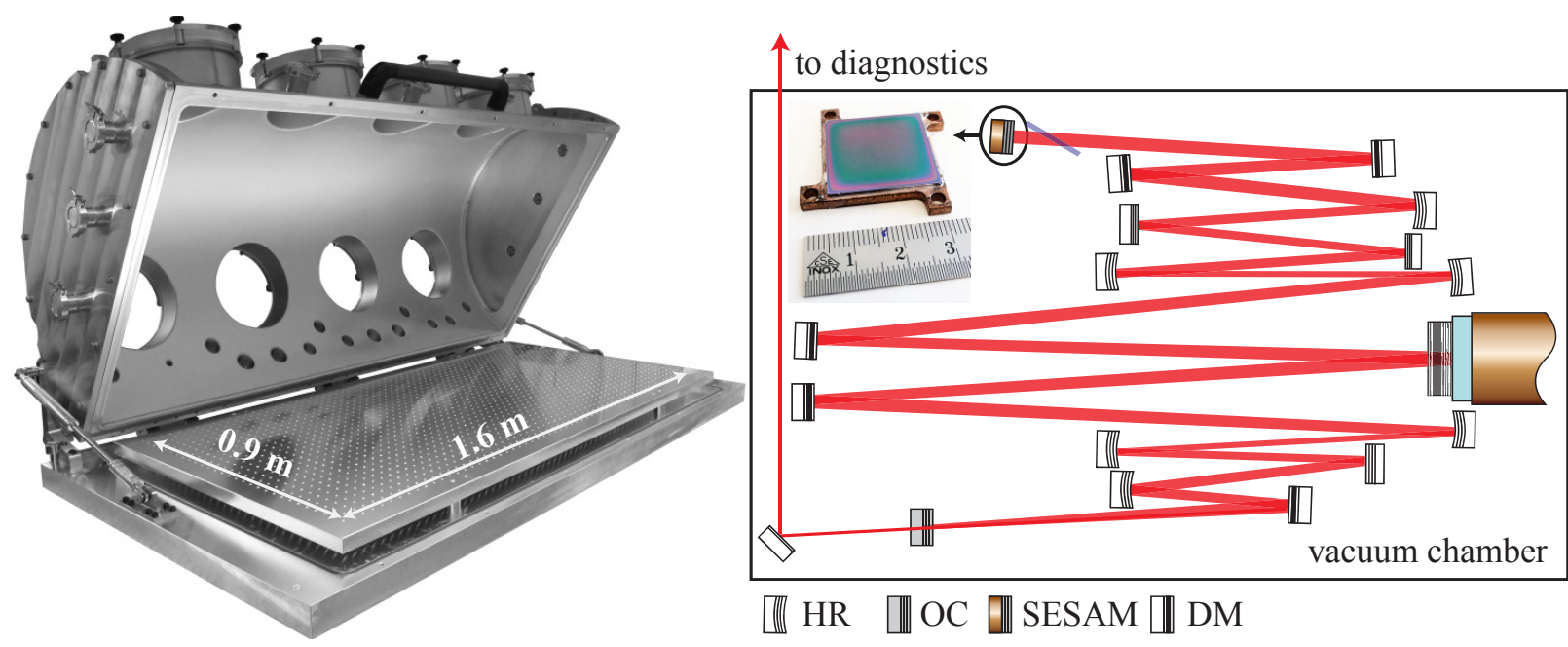

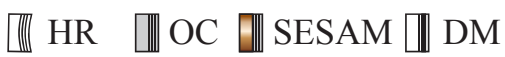


Figure 5. (a) Autocorrelation trace of the pulses at the maximum average output power of $275 \mathrm{~W}$; (b) Optical spectrum of the pulses; (c) Radio frequency spectrum of the pulses, with a resolution bandwidth of $30 \mathrm{kHz}$; (d) Output power and optical-to-optical efficiency in modelocked operation as a function of pump power. The points before modelocking were not measured in order to avoid damage of intracavity components during the Q-switched modelocking (QML) regime that occurs before modelocked operation.

(a)

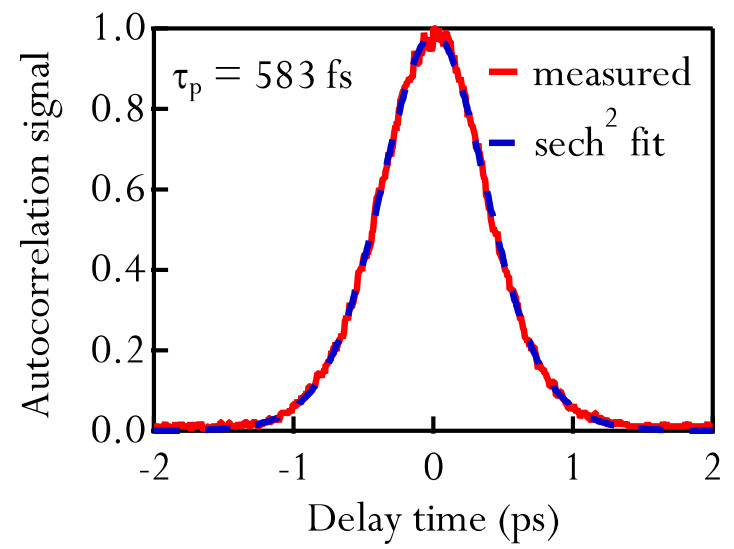

(c)

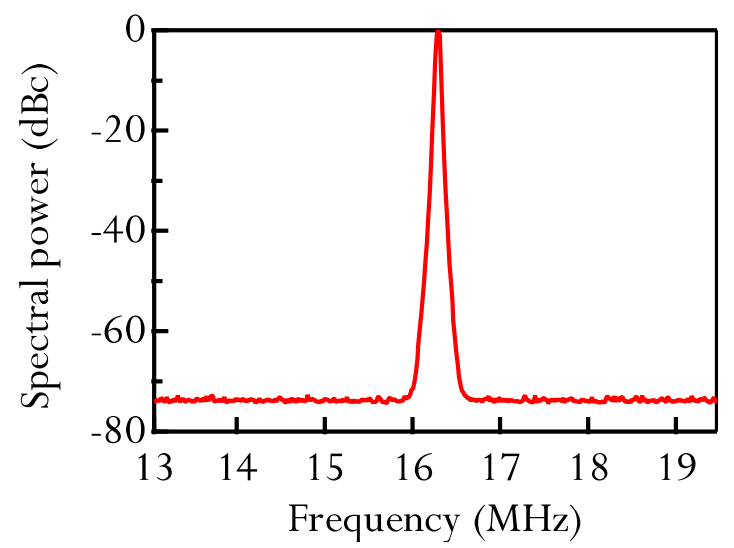

(b)

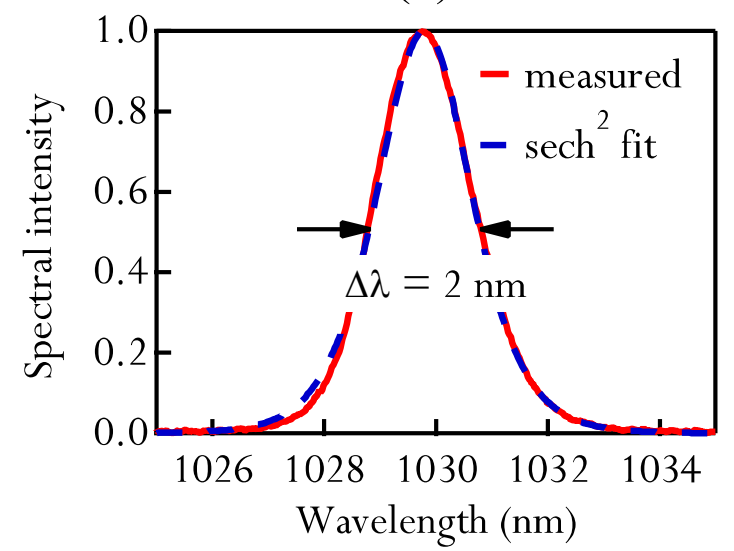

(d)

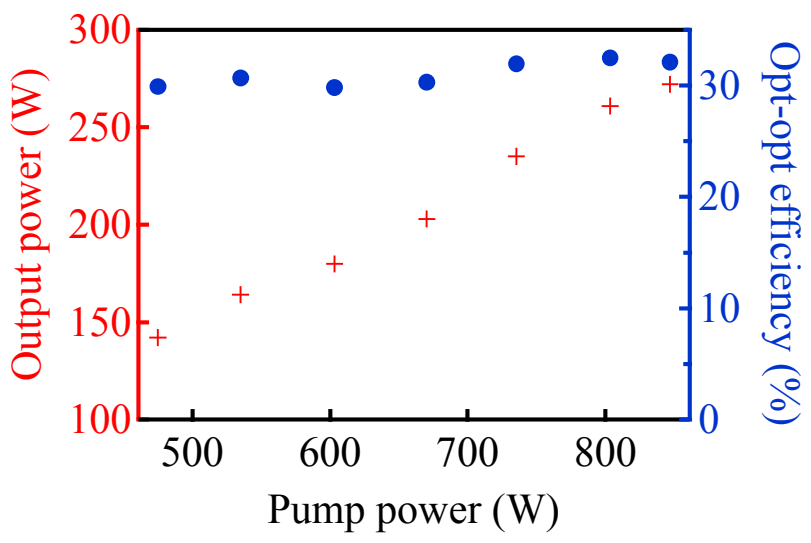

\subsubsection{Discussion}

Inside the oscillator, the circulating pulses had an energy of $146 \mu \mathrm{J}$, and a peak power of $220 \mathrm{MW}$. In contrast to previous high-power modelocked TDLs, the nonlinearity of the ambient environment was not the main contribution to the total SPM phase shift (Table 1). Assuming soliton pulses, the dispersion introduced in our cavity compensates for a nonlinear phase shift of approximately $75 \mathrm{mrad}$ at the maximum power and for the obtained pulse duration. The atmosphere in the cavity only contributes $\approx 8 \mathrm{mrad}$ to this total phase shift, assuming a linear behavior of the nonlinear refractive index of air with the pressure in the vacuum chamber [35,39]. The Brewster plate accounts for $\approx 17 \mathrm{mrad}$ and the thin disk for $\approx 3 \mathrm{mrad}$. The remaining phase shift ( $\approx 47 \mathrm{mrad}$ ) seems to originate from nonlinearities due the high intensities on the different cavity mirrors. In our current layout, some of the dielectric mirrors used in the cavity withstand intensities $>50 \mathrm{GW} / \mathrm{cm}^{2}$. At these high peak intensities, even a small penetration depth can lead to a significant phase shift. However, in order to precisely evaluate 
the contribution of each mirror to the total phase shift, the exact structure and material composition of these commercial mirrors needs to be precisely known. This point is currently being investigated.

In spite of these very high intracavity intensities, no damage was observed on the SESAM. The main limitation to higher average powers in our current configuration was thermal effects and even damage that occurred in the dispersive mirrors. Improved dispersive mirror designs with better thermal properties will allow for higher average power in the future.

\subsection{SESAMs for High-Power Femtosecond Modelocking}

\subsubsection{SESAMs in High-Power Ultrafast Oscillators}

As we mentioned in the previous paragraph, SESAMs with high-damage threshold and appropriate macroscopic parameters are one of the key points for power scaling of ultrafast oscillators. A specific investigation of damage and lifetime of SESAMs designed for high-power oscillators is therefore crucial. Previous investigations on optimized SESAM designs mostly focused on the realization of low saturation fluences [40,41], which is key for high repetition rates and stable modelocking of semiconductor lasers [34]. A recent investigation carried out in the context of high-energy modelocked TDLs focused on studying the influence of nitrogen incorporation on the carrier dynamics of SESAMs, but damage was not investigated [42].

SESAMs for high-power ultrafast TDLs operate in a regime in which pulse energies and average power levels are several orders of magnitude higher than in standard low-power femtosecond oscillators. In Table 2, we present typical SESAM operation parameters in recently demonstrated high-power thin-disk oscillators. We notice that SESAMs in such oscillators operate at kilowatt intracavity power levels, peak intensities of gigawatts per square centimeter, and fluences above the millijoule per square centimeter level. The saturation parameter ( $S$-parameter, $S=F / F_{\text {sat }}$ ) is a useful parameter to describe operation of a SESAM in a laser cavity. Typical SESAM modelocked lasers operate at $S=3-10$ [29], but, as we can see from Table 2, stable operation with $S$ parameters larger than 20 is common in high-energy oscillators. Operation at high $S$-parameters makes multi-pulsing instabilities a critical issue in TDLs. In particular, the additional absorption observed at high fluences caused by induced absorption (IA) causes a "rollover" in reflectivity. Operating the SESAM close to this rollover can lead to multi-pulsing instabilities [43-45] because in this case multiple pulses with lower pulse energy can have a gain advantage compared to single pulses.

Therefore, the most crucial parameters of SESAMs for high-power oscillators are:

- large saturation fluences to operate at moderate saturation parameters and with small spot sizes, which relaxes cavity sensitivity to alignment and possible thermal lensing,

- high damage thresholds,

- low nonsaturable losses to avoid thermal effects,

- reduced induced absorption (IA), which is responsible for the reflectivity rollover at high fluences and can lead to multi-pulsing instabilities [43-45].

The study presented in [19] focuses on how to tailor and combine suitable parameters for high-power operation and high-damage thresholds. As we have mentioned in paragraph 2.2, the latest average-power scaling step was made possible thanks to the guidelines established during this investigation. 
Table 2. Typical operation parameters of SESAMs in state-of-the-art high-power TDLs.

\begin{tabular}{llll}
\hline Parameter & Yb:YAG [17] & Yb:YAG [18] & Yb:LuO [26] \\
\hline Average output power & $275 \mathrm{~W}$ & $145 \mathrm{~W}$ & $141 \mathrm{~W}$ \\
Repetition rate & $16.3 \mathrm{MHz}$ & $3.5 \mathrm{MHz}$ & $60 \mathrm{MHz}$ \\
Pulse duration & $583 \mathrm{fs}$ & $1100 \mathrm{fs}$ & $740 \mathrm{fs}$ \\
Output pulse energy & $16.9 \mu \mathrm{J}$ & $41 \mu \mathrm{J}$ & $2.35 \mu \mathrm{J}$ \\
Output peak power & $25.6 \mathrm{MW}$ & $33 \mathrm{MW}$ & $2.8 \mathrm{MW}$ \\
\hline Intracavity average power & $2.5 \mathrm{~kW}$ & $0.2 \mathrm{~kW}$ & $1.5 \mathrm{~kW}$ \\
Intracavity pulse energy & $154 \mu \mathrm{J}$ & $57 \mu \mathrm{J}$ & $25 \mu \mathrm{J}$ \\
Intracavity peak power & $236 \mathrm{MW}$ & $46 \mathrm{MW}$ & $30 \mathrm{MW}$ \\
\hline Spot radius on SESAM $\left(1 / \mathrm{e}^{2}\right)$ & $1.2 \mathrm{~mm}$ & $0.55 \mathrm{~mm}$ & $0.63 \mathrm{~mm}$ \\
Fluence on SESAM & $3.4 \mathrm{~mJ} / \mathrm{cm}^{2}$ & $6 \mathrm{~mJ} / \mathrm{cm}^{2}$ & $2 \mathrm{~mJ} / \mathrm{cm}^{2}$ \\
Average intensity on SESAM & $55 \mathrm{~kW} / \mathrm{cm}^{2}$ & $21 \mathrm{~kW} / \mathrm{cm}^{2}$ & $12 \mathrm{~kW} / \mathrm{cm}^{2}$ \\
Peak intensity on SESAM & $5.2 \mathrm{GW} / \mathrm{cm}^{2}$ & $4.8 \mathrm{GW} / \mathrm{cm}^{2}$ & $5 \mathrm{GW} / \mathrm{cm}^{2}$ \\
\hline Saturation fluence of SESAM & $140 \mu \mathrm{J} / \mathrm{cm}^{2}$ & $61 \mu \mathrm{J} / \mathrm{cm}^{2}$ & $60 \mu \mathrm{J} / \mathrm{cm}^{2}$ \\
Saturation parameter $\left(S=F / F_{\text {sat }}\right)$ & 24 & 100 & 33 \\
\hline
\end{tabular}

\subsubsection{Experimental Setup and Measurement Procedure}

In order to reach the high fluences necessary to carry out this study, we used a high-energy SESAM modelocked Yb:YAG TDL seeding a high-precision nonlinear reflectivity measurement setup to characterize nonlinear reflectivity, IA, and damage of our SESAMs. The experimental setup is presented in Figure 6, and in more detail in reference [46]. The SESAM modelocked Yb:YAG TDL delivers $15 \mathrm{~W}$ of average power at a repetition rate of $10.7 \mathrm{MHz}$, corresponding to a pulse energy of $1.4 \mu \mathrm{J}$ in 1-ps pulses, which allowed testing SESAMs up to an unprecedentedly high fluence of $0.21 \mathrm{~J} / \mathrm{cm}^{2}$.

Figure 6. (a) Experimental setup used for the nonlinear reflectivity characterization and damage measurements; (b) Typical measurement of nonlinear reflectivity measured using this setup, and important macroscopic parameters extracted using a least-squares fitting procedure [47].

(a)

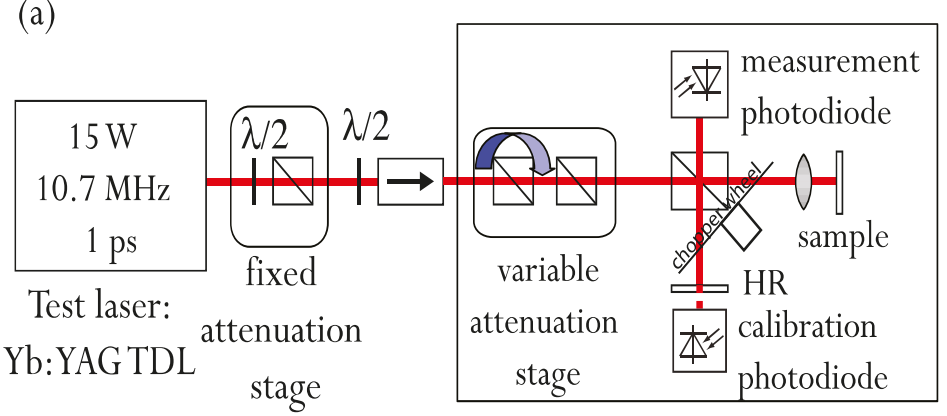

Nonlinear reflectivity setup

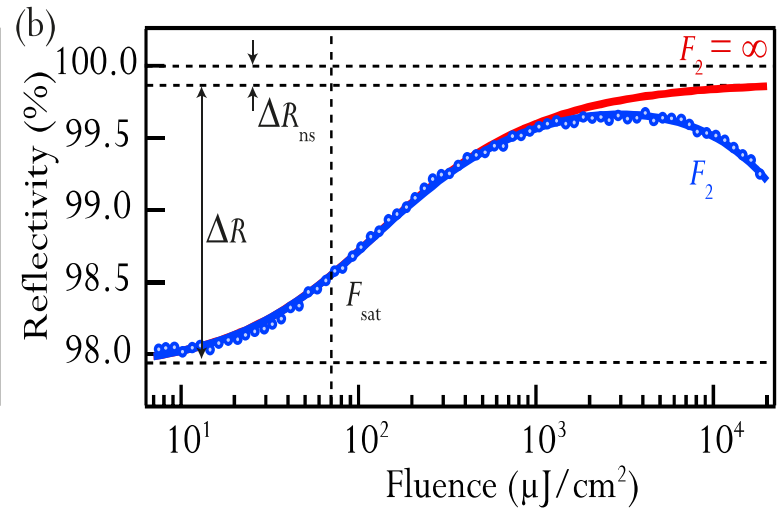


Formula (4) describes the fluence-dependent SESAM reflectivity $R(F)$ for a flat-top-shaped beam profile [47]:

$$
R(F)=R_{\mathrm{rs}} \frac{\ln \left(1+\frac{R_{\text {in }}}{R_{\mathrm{rs}}}\left(\mathrm{e}^{\frac{F}{F_{\text {st }}}}-1\right)\right)}{\frac{F}{F_{\text {sd }}}} \mathrm{e}^{-\frac{F}{\frac{F}{2}}}
$$

A typical measurement and the important extracted macroscopic parameters using this formula with a numerical correction for a Gaussian beam profile are presented in Figure 6. These parameters are:

- The modulation depth $\Delta R$, which represents the maximum achievable change in reflectivity.

- The nonsaturable losses $\Delta R_{\mathrm{ns}}$ represent the unsaturable fraction of the reflectivity which originate from defect absorption, scattering, free-carrier absorption, carrier heating, etc.

- The saturation fluence $F_{\text {sat }}$ represents, in the case of moderate modulation depths (up to approximately $10 \%$ ), the fluence at which $1 / \mathrm{e}$ of the modulation depth has been saturated.

- The IA coefficient $F_{2}$, which characterizes the strength of the reflectivity rollover that occurs at high fluences. In the case of femtosecond pulses, two-photon absorption (TPA) is the main cause of IA $[19,45]$. However, for longer pulse durations, studies show that the measured rollover is stronger than predicted by TPA only, indicating that other effects need to be considered, such as free-carrier absorption, or hot-carrier generation $[43,45,48]$.

In addition to the nonlinear reflectivity parameters, this setup was used to measure the damage fluence and lifetime of different representative SESAMs. In this study, damage was defined as an irreversible change in the structure resulting in a dramatic drop in the measured reflectivity. The damage fluence threshold $F_{\mathrm{d}}$ is then defined as the minimum fluence where this irreversible reflectivity drop occurs in $<1 \mathrm{~s}$. In order to measure the damage fluence and the time-to-damage of a sample we use the same setup described in the first section for nonlinear reflectivity measurements. We set the fluence to a constant value and track reflectivity of the sample versus time. In this way, we can measure the lifetime of different samples by evaluating the time-to-damage at fluences lower than the damage fluence.

\subsubsection{Studied Structures and Obtained Results}

A common approach to increase the saturation fluence of a SESAM consists of growing a top mirror on the structure to increase its finesse. In this way, the electric field in the absorber layers is reduced and the fluence required to saturate the sample is increased. As we increase the saturation fluence, we reduce the modulation depth of the sample by the same factor, since $F_{\text {sat }} \Delta R=F_{\mathrm{t}}$ with $F_{\mathrm{t}}$ the transparency fluence of the absorber. The product $F_{\text {sat }} \Delta R$ remains constant for a given absorber section since the transparency fluence only depends on intrinsic material properties [19,49]. This means that the samples to topcoat need to have a large enough modulation depth in addition to the basic requirements (i.e., low nonsaturable losses and an initially large saturation fluence). With multiple QWs, we can adjust the modulation depth without changing the saturation fluence of the samples.

The design of the non-topcoated SESAM (NTC) used for this study consists of a 30-pair GaAs/AlAs DBR and three 10-nm InGaAs QWs as absorber layers in an antiresonant configuration. 
The QWs of this sample were grown at $T \approx 400{ }^{\circ} \mathrm{C}$, resulting in a recovery time at $1 / \mathrm{e}$ of $\tau_{1 / \mathrm{e}} \approx 200 \mathrm{ps}$ and low nonsaturable losses $<0.1 \%$.

Three different topcoatings were grown on this structure. In the first case, a semiconductor topcoating was chosen with four pairs of quarter-wave GaAs/AlAs layers (semiconductor topcoating (SCTC), see Figure $7 b$ ) grown by molecular beam epitaxy (MBE). In this design, the field enhancement in the absorber is reduced by a factor of $\approx 4$ compared to the uncoated sample (see Figure $7 \mathrm{~d}$ ). Therefore the same increase of the saturation fluence is expected. For the other cases, a dielectric $\mathrm{SiO}_{2} / \mathrm{Si}_{3} \mathrm{~N}_{4}$ topcoating deposited by plasma-enhanced chemical vapor deposition (PECVD) was chosen. Two sets of samples were coated with two and three pairs of quarter-wave layers (DTC2 and DTC3, see Figure 7c). In this case, we expect an increase of the saturation fluence by a factor of 3 and 5 , respectively Figure $7 \mathrm{~d}$. The dielectric topcoating can be applied after MBE growth, which allows for additional flexibility in terms of finesse change of the structure. From a material point of view, $\mathrm{SiO}_{2}$ and $\mathrm{Si}_{3} \mathrm{~N}_{4}$ are dielectric materials, and therefore exhibit negligible TPA compared to GaAs [50], which results in reduced IA.

Figure 7. Different SESAM structures used for the damage studies (a) SESAM design with 3 QWs and no topcoating (NTC); (b) same SESAM but with a 4-quarter-wave-pair GaAs/AlAs semiconductor topcoating (SCTC); (c) same SESAM but with a 3-quarter-wave pair $\mathrm{SiO}_{2} / \mathrm{Si}_{3} \mathrm{~N}_{4}$ dielectric topcoating (DTC3); (d) field enhancement in the absorber section.

(a)

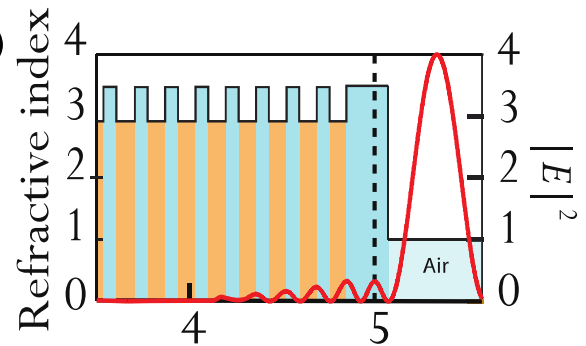

(b)

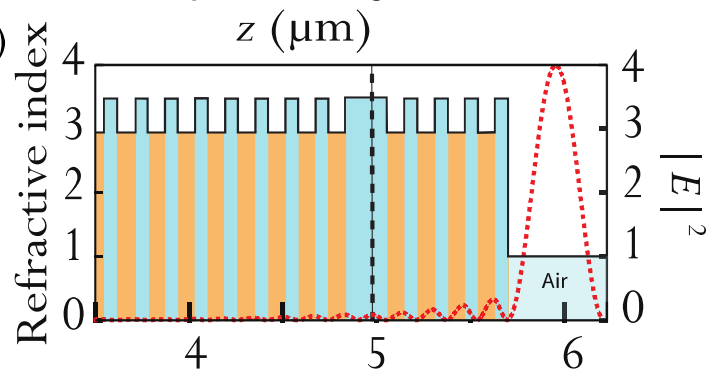

(c)

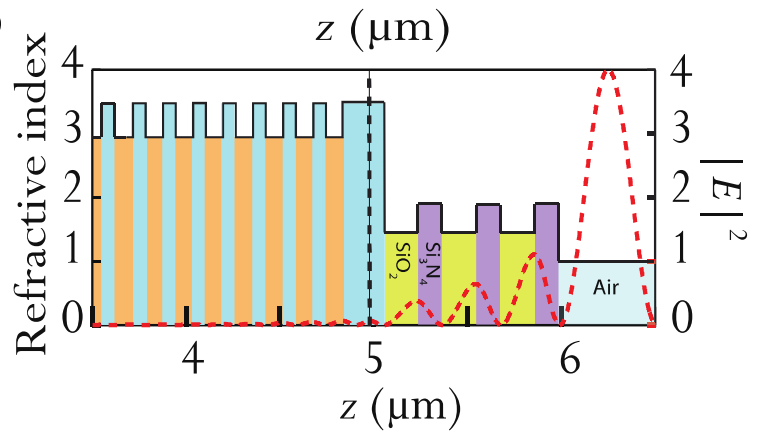

(d)

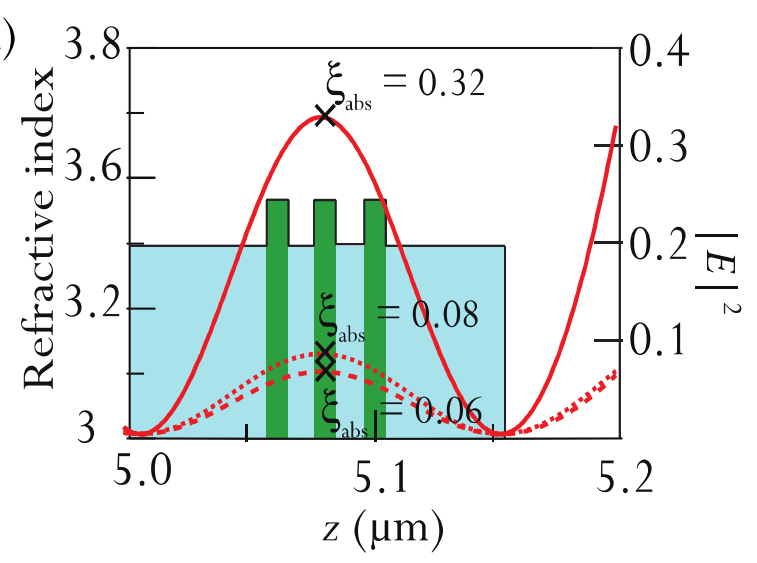

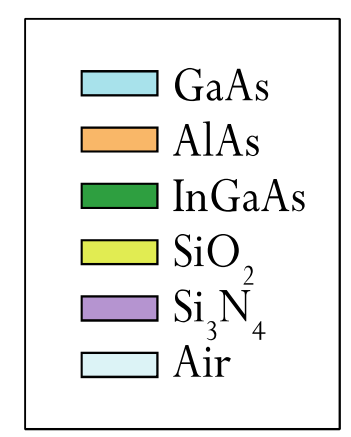


Figure 8. (a) Damage threshold (indicated with a star) of the different tested SESAMs together with their nonlinear reflectivity measurement; (b) Lifetime curves of different representative SESAMs, where one can see a large shift of the lifetime of the sample with a dielectric topcoating to higher fluences. The sample with a 3-pair dielectric topcoating (DTC3) could not be damaged at the maximum available fluence in our setup of $0.21 \mathrm{~J} / \mathrm{cm}^{2}$.
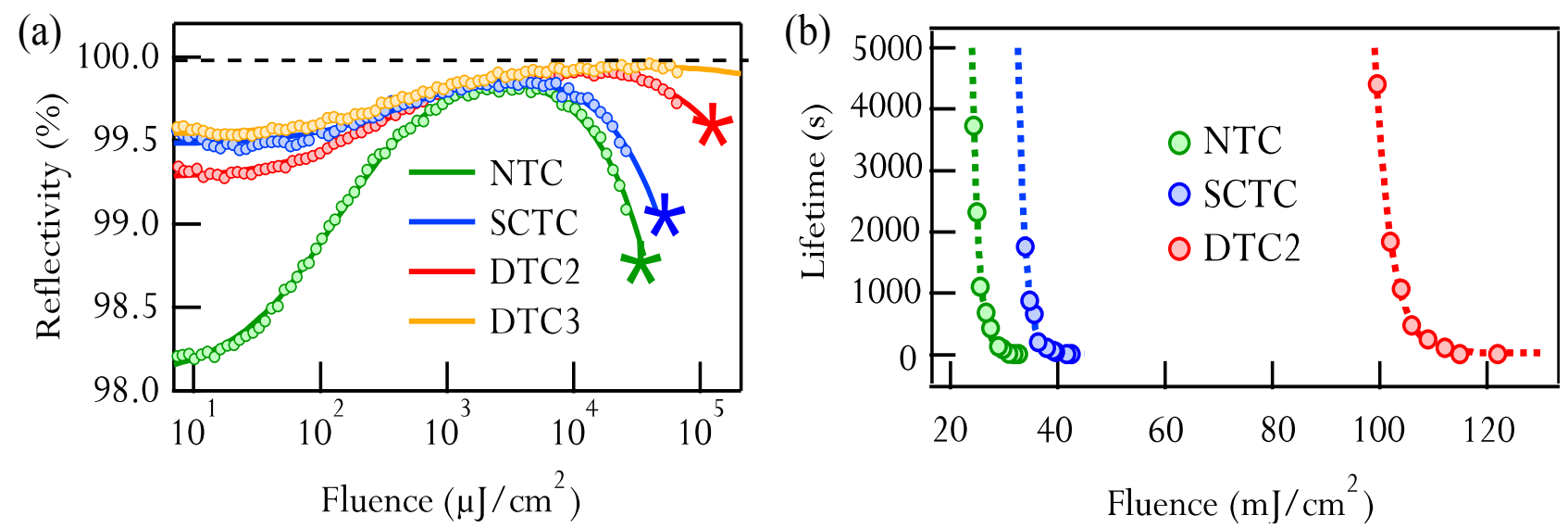

The nonlinear reflectivity measurements and corresponding extracted parameters are presented together with the measured damage thresholds in Figure 8a, and Table 3. We can clearly see the effect of the different topcoatings on the saturation parameters of the samples: as the saturation fluence increases, the modulation depth decreases by a similar factor. The saturation fluences of all the topcoated samples are larger than $150 \mu \mathrm{J} / \mathrm{cm}^{2}$ and their modulation depths are between $0.4 \%$ and $0.8 \%$. The 3-QW non-topcoated sample with 2\% modulation depth is ideally suited for topcoatings designed for a saturation fluence increase of 3-5. All SESAMs have negligible nonsaturable losses $<0.1 \%$ and therefore minimal thermal load.

Table 3. Damage thresholds of the different representative SESAMS together with their saturation parameters. The samples with a 3-pair dielectric topcoating did not show damage up to the maximum available fluence in our setup of $0.21 \mathrm{~J} / \mathrm{cm}^{2}$.

\begin{tabular}{ccccccc}
\hline Sample & $\begin{array}{c}\boldsymbol{F}_{\text {sat }} \\
\left(\boldsymbol{\mu J} / \mathbf{c m}^{2}\right)\end{array}$ & $\boldsymbol{\Delta} \boldsymbol{R}(\boldsymbol{\%})$ & $\boldsymbol{\Delta} \boldsymbol{R}_{\mathrm{ns}}(\mathbf{\%})$ & $\begin{array}{c}\boldsymbol{F}_{\mathbf{2}} \\
\left(\mathbf{m J} / \mathbf{c m}^{2}\right)\end{array}$ & $\boldsymbol{F}_{\mathrm{d}}\left(\mathbf{m J} / \mathbf{c m}^{2}\right)$ & $\boldsymbol{S}_{\mathrm{d}}=\boldsymbol{F}_{\mathrm{d}} / \boldsymbol{F}_{\text {sat }}$ \\
\hline NTC & 72 & 2.05 & $<0.1$ & 3200 & 32.6 & 450 \\
SCTC & 279 & 0.52 & $<0.1$ & 5500 & 44.1 & 158 \\
DTC2 & 168 & 0.71 & $<0.1$ & 31700 & 122 & 726 \\
DTC3 & 247 & 0.43 & $<0.1$ & 346000 & $>210$ & $>850$ \\
\hline
\end{tabular}

In terms of IA, all topcoated SESAMs have increased $F_{2}$ coefficients compared to that of the non-topcoated sample. However, the semiconductor topcoated SESAM (SCTC) shows only a small increase compared to the dielectric topcoated samples (DTC2 and DTC3). Although the field in the DBR structure and the absorber section is reduced in all samples, the GaAs/AlAs topsection experiences a strong electric field, and GaAs has a strong TPA coefficient. In the case of the dielectric topcoating, both materials have a negligible TPA coefficient compared to GaAs. Therefore, SESAMs with similar saturation parameters (similar electric field distributions in the DBR and the absorber region) but different topcoatings have different IA responses. 
The topcoated SESAMs show, in all cases, higher damage fluences than without a topcoating. The damage fluences for the dielectric topcoated SESAMs (DTC2 and DTC3) are much higher than for the semiconductor topcoated SESAM (SCTC) with similar saturation parameters. It is interesting to note that regardless of the sample, instantaneous damage occurs at fluences deep in the rollover regime, where SESAM modelocked lasers would not operate in a stable regime (e.g., saturation parameters larger than 150). However, the higher damage threshold is beneficial to overcome the Q-switched modelocking (QML) regime usually observed before modelocking [51], regime where peak powers can be significantly larger than in stable cw-modelocking. Furthermore, the lifetime of these samples is longer, which is of interest for long-term operation. For our sample with 3 quarter-wave pairs dielectric topcoating (DTC3), damage was not observed even at the maximum available fluence in our setup of $0.21 \mathrm{~J} / \mathrm{cm}^{2}$. This particular sample was tested at this maximum fluence level for several hours and no damage was observed.

Lifetime curves were measured in this high-fluence regime (Figure 8b), showing a clear exponential behavior for all samples. This suggests lifetimes of several 10,000 hours at standard operation parameters. However, it is likely that other mechanisms need to be taken into account to correctly evaluate the lifetime at much lower fluences.

\subsubsection{Damage Mechanism}

The damage fluence measurements presented in the previous paragraph suggest a damage mechanism related to the absorbed energy due to IA. It is interesting to note that in the case of the sample with 3 QWs and a two-pair dielectric topcoating (DTC2) where $F_{2}$ is greatly increased, the damage curve is also shifted to higher values. In order to confirm this point, the damage behavior of a SESAM with a single QW absorber, one with 3 QWs, and a DBR mirror (without absorber section) were compared (Figure 9 and Table 4). This is crucial to evaluate if the damage threshold is dependent on the absorber geometry. All the samples have no topcoating, and were grown in an antiresonant configuration.

Figure 9. Nonlinear reflectivity and lifetime measurements for a (distributed Bragg reflector) (DBR), a SESAM with $1 \mathrm{QW}$ as an absorber and a similar SESAM with 3 QWs as absorbers.

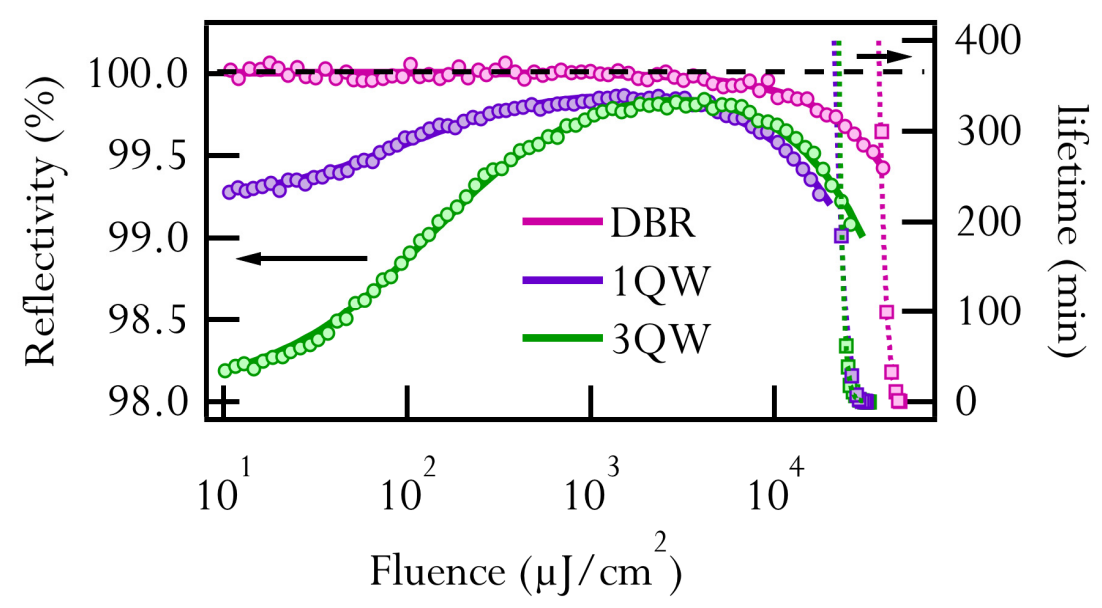


Table 4. Relevant parameters and damage thresholds of the samples with different absorber sections.

\begin{tabular}{lcccc}
\hline Sample & $\boldsymbol{F}_{\text {sat }}\left(\boldsymbol{\mu} \mathbf{J} / \mathbf{c m}^{\mathbf{2}}\right)$ & $\boldsymbol{F}_{\mathbf{2}}\left(\mathbf{m J} / \mathbf{c m}^{\mathbf{2}}\right)$ & $\boldsymbol{F}_{\mathrm{d}}\left(\mathbf{m J} / \mathbf{c m}^{\mathbf{2}}\right)$ & $\boldsymbol{S}_{\mathbf{d}}=\boldsymbol{F}_{\mathrm{d}} / \boldsymbol{F}_{\text {sat }}$ \\
\hline DBR & - & 7400 & 48 & - \\
1 QW & 57 & 2600 & 32.1 & 560 \\
3 QW & 72 & 3200 & 32.6 & 453 \\
\hline
\end{tabular}

We can see (Figure 9 and Table 4) that the damage behavior of a DBR is similar to that of the characterized SESAMs. Damage occurs deep in the rollover regime, and this rollover occurs at comparable fluence levels as for all tested SESAMs. The measurements confirm that the damage mechanism is related to the absorbed energy due to IA. This fraction of absorbed energy per area $F_{\text {abs }}$ due to the IA can be evaluated at in incident fluence $F$ taking into account a number of approximations [19] by:

$$
F_{\mathrm{ds}} \approx \frac{F}{F_{2}}
$$

Therefore, the damage fluence $F_{\mathrm{d}}$ for all SESAMs should scale proportionally to $\sqrt{F_{2}}$. In order to illustrate this dependence, we plotted the ratio $\sqrt{ } F_{2} / F_{\mathrm{d}}$ for 16 different samples for which we were able to measure the damage fluence (Figure 10).

Figure 10. Ratio $\sqrt{ } F_{2} / F_{\mathrm{d}}$ for different samples for which we measured the damage threshold, including samples with different absorber sections and a DBR mirror.

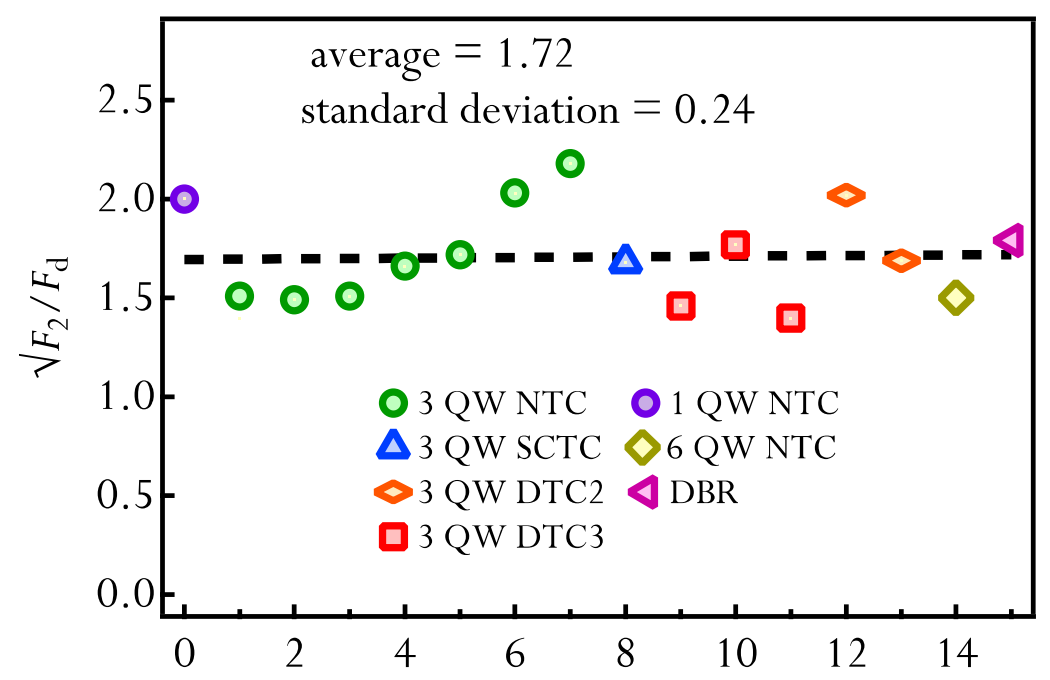

The data seems to confirm that the main contribution to IA and damage originates in the field in the DBR and spacer layers and not by the absorber section itself. This gives a clear indication of the damage behavior of such SESAMs, suggesting that catastrophic damage occurs due to heating of the lattice by energy absorbed by the IA process. It also indicates how to shift the damage fluence to higher values by simply increasing $F_{2}$. 


\subsubsection{Influence of Growth Temperature}

In some specific cases, additional requirements on the SESAM parameters can be beneficial to push the oscillator performance to its limits. For example, in the case of TDLs where short pulses are targeted and a large fraction of the bandwidth needs to be exploited, a somewhat higher modulation depth and faster recovery time are beneficial [20,28,29]. Achieving shorter recovery times is straightforward with low-temperature (LT) growth [52]. The results presented in the previous paragraph indicate that growing the QWs at lower temperature should only have a small influence on the damage threshold of the samples, since the damage threshold is nearly independent of the absorber section. However, an experimental verification of this point is crucial, in particular given the higher nonsaturable losses expected from such LT-grown QW-SESAMs. In this paragraph, we will present measurements that confirm that the growth temperature of the QWs has only a small influence on the damage threshold, indicating that the guidelines presented in the previous paragraph can also be applied to fast samples with non-negligible nonsaturable losses.

\section{- Experimental Setup}

In order to carry out this study, we measured the nonlinear reflectivity, damage threshold and recovery parameters of a set of representative samples. For the nonlinear reflectivity and the damage threshold measurements, we used the same setup and experimental procedure described in 2.3.2. To measure the recovery time of the samples, we used a standard pump-probe setup (Figure 11) seeded by a bulk Yb:YAG laser delivering 1-ps long pulses at a repetition rate of $38 \mathrm{MHz}$ and $150 \mathrm{~mW}$ of average power. The laser operates at a central wavelength of $1030 \mathrm{~nm}$. For the study of the influence of the absorber growth temperature, we used samples with a larger number of QWs than in the damage and lifetime investigation presented in the previous paragraph in order to reach an initially larger modulation depth. The structure consists of a standard DBR and four InGaAs QW absorbers embedded in GaAs. The absorbers were placed two-by-two in two consecutive antinodes of the electric field pattern to optimize their absorption. This resulted in samples with more than $3 \%$ modulation depth, which can be further coated to decrease their modulation depth. The QW absorbers of the four samples used for this study were grown at different temperatures $\left(245{ }^{\circ} \mathrm{C}, 270{ }^{\circ} \mathrm{C}, 300{ }^{\circ} \mathrm{C}, 385{ }^{\circ} \mathrm{C}\right)$. The samples were designed for an operation wavelength of $1030 \mathrm{~nm}$.

- Results

In Figure 12a, pump-probe measurements of these samples are shown, confirming as expected that samples grown at lower temperatures have faster recovery times [52]. In Figure 12b, we plot the characteristic decay time to $1 / \mathrm{e}\left(\tau_{1 / \mathrm{e}}\right)$ as a function of QW growth temperature. The observed behavior confirms previous studies carried out with LT-grown GaAs [52]. Although the parameter $\tau_{1 / \mathrm{e}}$ is not sufficient to fully characterize the dynamics of the absorber, it is an appropriate simplified parameter to compare the recovery of the absorber in a passively modelocked solid-state laser. In Figure 12c, we plot nonsaturable losses and damage threshold of these samples as a function of the growth temperature. The total nonsaturable losses of the samples decrease exponentially with the growth temperature, which also confirms the observations made in reference [52]. It is interesting to point out that even for our fastest sample with a 
recovery time of $\approx 2$ ps the nonsaturable losses stay low in comparison to the modulation depth of the sample (1.2\% nonsaturable losses for 3.9\% modulation depth). For use in most common TDLs, lower modulation depth is required. Therefore a dielectric topcoating that will reduce the field in the absorber will be applied, further reducing the nonsaturable losses. The measured increase in damage threshold with growth temperature is relatively small compared to the decrease in the recovery time of the absorber (we observe a decrease of $40 \%$ in damage threshold, for a 12 -fold decrease of the nonsaturable losses). Heating of the absorbers due to the additional defects most likely causes this small decrease in the damage threshold of the samples.

Figure 11. Pump-probe setup used for measuring the recovery dynamics of the different SESAMs. AOM: Acousto-optic modulator.

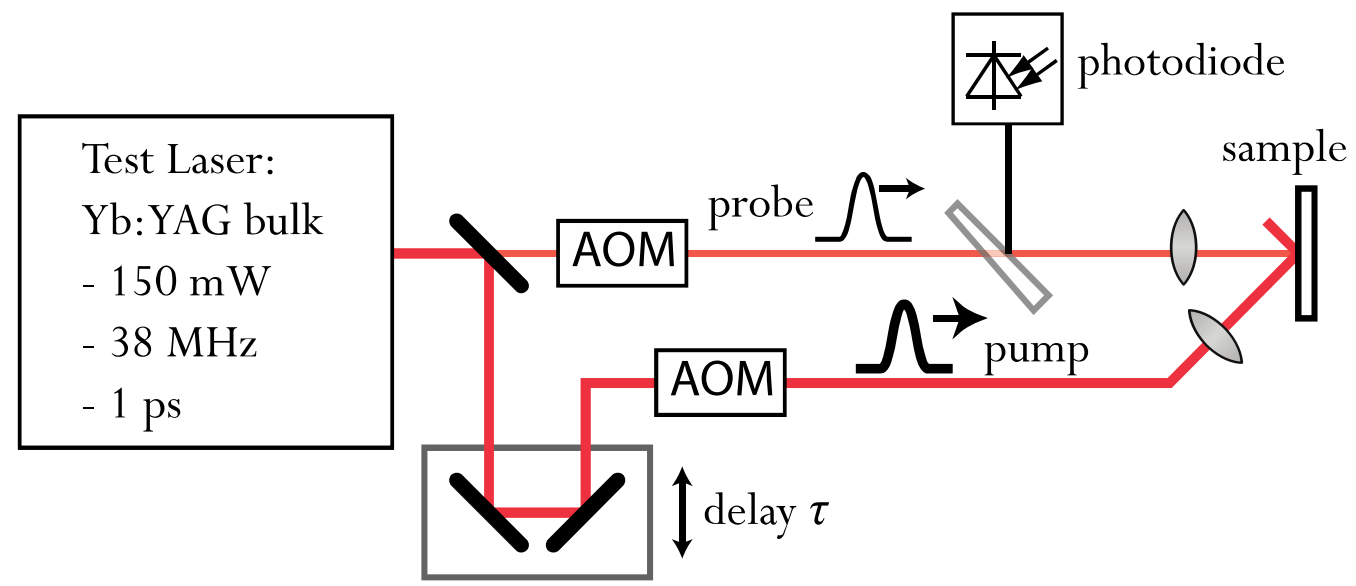

Figure 12. (a) Pump-probe measurements of samples with the same structure, but with the absorbers grown at different temperatures; (b) Dependence recovery time at 1/e on absorber growth temperature; (c) Dependence of damage threshold (green) and nonsaturable losses (red) on QW growth temperature.

(a)

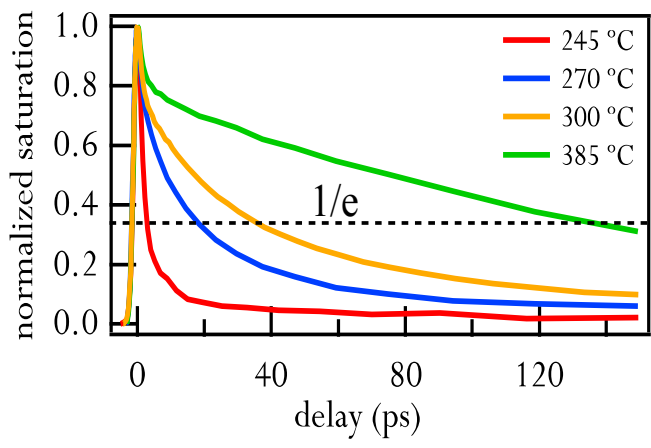

(b)

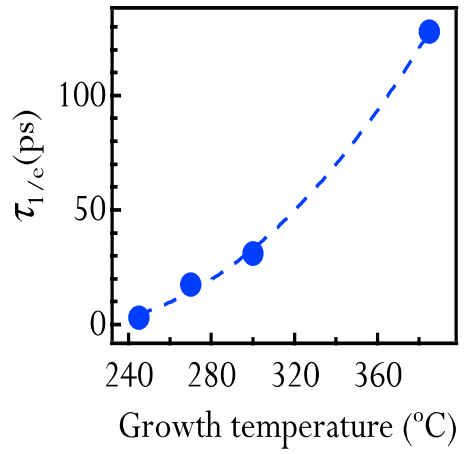

(c)

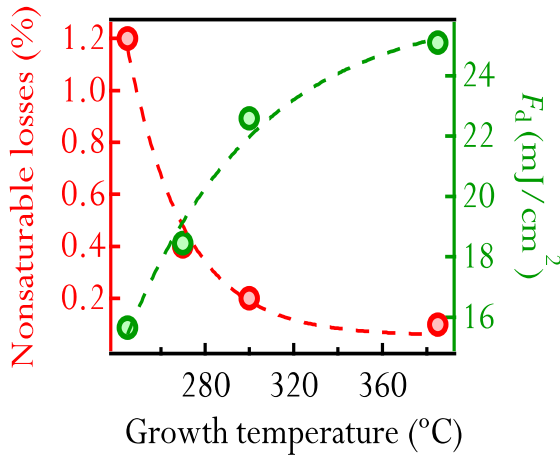

These measurements indicate that the nonsaturable losses introduced by the additional defects in the absorbers also contribute to the damage mechanism. However, this influence is small relative to the change in the recovery time of the samples. Furthermore, even for our fastest samples, damage occurs at fluence levels where modelocked lasers do not operate in a stable configuration. 


\subsubsection{Guidelines and Outlook}

- Guidelines for high-damage threshold SESAMs

In this study, important elements concerning the catastrophic damage of SESAMs that occurs at high fluences were identified. On the one hand, damage originates in the amount of energy deposited by IA on the sample. Therefore, damage threshold scales proportionally to $\sqrt{F_{2}}$. In our study, we used 1-ps long pulses. The dominant mechanism involved in the IA (and therefore damage) at this pulse duration is TPA [45]. On the other hand, the damage threshold was shown to be nearly independent of the absorber section, since a DBR showed a comparable damage threshold and lifetime behavior to those of the different tested SESAMs. As a result, we can give guidelines to develop SESAMs for operation in high-power oscillators:

- Multiple QWs allow for tuning of the modulation depth of the samples without changing their saturation fluence. This is required to have an initially large $\Delta R$ without topcoating, since the top mirror will reduce this modulation depth. When a relatively low number of QWs is used, the absorbers can be placed simultaneously in one antinode of the electric field. In this case, the absorbers have only a small influence on the damage threshold, mostly because of the additional GaAs spacer layers commonly used as barrier material. When a larger number of QW is used, the amount of material to add becomes significant. In this case, one could also consider using another material with lower $\beta_{\text {TPA }}$ (for instance AlAs) for such QW barrier layers. Without GaAs barrier layers, we expect uncoated SESAM samples to have an almost identical damage behavior to that of a DBR mirror.

- Dielectric topcoatings are preferred over semiconductor topcoatings to increase the saturation fluence. The topsection of a SESAM is critical because it experiences a very strong electric field and therefore contributes strongly to the IA and to the damage. With a dielectric material, we achieve larger values for $F_{2}$ and, consequently, higher damage fluences.

- The absorber section of the samples used for the damage and lifetime study was grown at $T \approx 400{ }^{\circ} \mathrm{C}$ leading to recovery times in the order of $\approx 200 \mathrm{ps}$ and negligible nonsaturable losses. Lower growth temperatures result in shorter recovery times but also in a larger growth defect densities and higher nonsaturable losses. Additional measurements show that the growth temperature only has a small influence on the damage threshold, which confirms the mechanism identified in this study.

- Future SESAM designs

- More QWs, more topcoating layers

The simple guidelines developed in this study indicate that multiplying the number of QWs and the number of dielectric layers results in high-damage threshold samples with reduced IA and high saturation fluences, which are all crucial parameters for high-power oscillators. However, the limits of this approach for example in terms of maximum amount of QWs and/or dielectric top layers were not investigated.

Concerning the dielectric topcoating, we chose the pair $\mathrm{SiO}_{2} / \mathrm{Si}_{3} \mathrm{~N}_{4}$ simply because the coatings could be applied in-house in just a few hours using PECVD. Most of the 
depositions were performed at $300{ }^{\circ} \mathrm{C}$ in order to achieve high optical quality layers. However, this deposition method has limits in terms of the number of layers that can be applied.

○ Stress: Given the high growth temperature of the PECVD topcoating, a large number of layers can result in significant stress of the coating at room temperature, which can lead to fracture. The limit in terms of number of layers can be increased up to some extent by reducing the deposition temperature, for example to $120^{\circ} \mathrm{C}$, at the expense of a slight degradation of the optical quality of the deposited coating. However, for a large number of layers, the use of other stress-less deposition techniques such as sputtering or evaporation should be considered.

- Materials: Different dielectric material pairs with a higher refractive index contrast are beneficial to reduce the necessary number of layers to achieve a given field enhancement in the absorbers. Some examples are $\mathrm{SiO}_{2} / \mathrm{Ta}_{2} \mathrm{O}_{5}$ (which is already commonly used as a topsection for SESAMs [32]), $\mathrm{SiO}_{2} / \mathrm{HfO}_{2}$ or $\mathrm{SiO}_{2} / \mathrm{TiO}_{2}$.

Using the maximum possible number of QWs in one antinode of the electric field is preferred to limit the amount of material used in the structure. However, the QWs need to be separated by a large enough distance such that they do not interact between each other. This sets a limit to the number of QWs in one antinode for achieving efficient absorption. Furthermore, the fabrication of SESAMs with large amount of QWs is challenging due to stress resulting from the difference in lattice constants of the absorbers and the spacer layers. Although the exact consequences of this stress on SESAM properties have not been studied in detail, strain-compensation methods might be necessary to maintain the losses in the sample low.

- Upside-down growth of large-scale SESAMs for improved thermal management Until now, thermal effects in SESAMs have not been the main limitation for power scaling of modelocked TDLs. Deposited heat in such structures is very low and is mainly due to the residual low nonsaturable losses of the samples. However, in future $\mathrm{kW}$ level oscillators, this small fraction of absorbed power can become significant, and lead to thermal aberrations and beam degradation [53]. The modelocked TDL power-scaling concept relies on increasing the spot sizes both on the disk and on the SESAM. Although for SESAMs we have the additional design freedom of increasing the saturation fluence to keep the saturation level constant, spot sizes will most likely also become larger as we move towards several $\mathrm{kW}$ of intracavity powers. One important consequence is that possible thermal lensing of the SESAM will then become a critical issue. Therefore, the design of large (several centimeters squared) SESAMs with improved thermal capabilities is a key point in this direction.

One possible improvement for better thermal management of SESAMs is substrate removal. In standard SESAMs, the DBR and the absorber section are grown in this order on a GaAs substrate. In this case, the GaAs substrate plays no optical role, and only limits heat transport due to its low thermal conductivity $\left(\kappa_{\mathrm{GaAs}} \approx 44 \mathrm{~W} \cdot \mathrm{m}^{-1} \cdot \mathrm{K}^{-1}\right)$. A common approach to improve the heat removal of the sample is to grow the structure upside down, contact the sample to a heatsink with a higher thermal 
conductivity (for example diamond $\kappa_{\text {diamond }} \approx 2200 \mathrm{~W} \cdot \mathrm{m}^{-1} \cdot \mathrm{K}^{-1}$ ) and remove the substrate. This technique is usually referred to as "flip-chip" bonding, and is most commonly used for power scaling of VECSELs [54-57], where the very high pump absorption makes this a more critical issue. In this way, heatsinks with better heat removal properties such as copper or diamond can be chosen. The resulting structure is very thin (typically a few $\mu \mathrm{m}$ thick) and can be very efficiently cooled. This technique is illustrated in Figure 13 for a metal heatsink (typically copper).

Figure 13. Schematic of the fabrication steps of future generation upside-down high-power SESAMs.

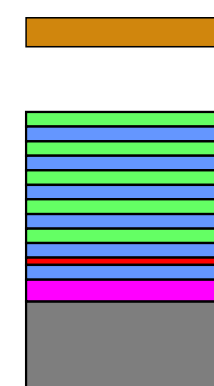

upside-down MBE growth, choice of substrate

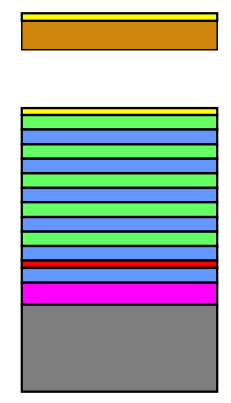

metalization (substrate and sample)

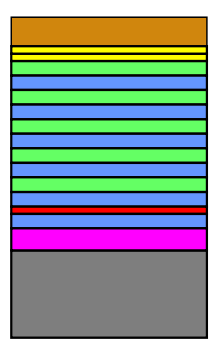

contacting

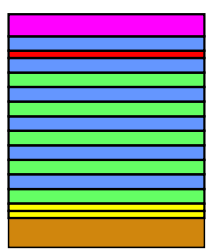

etching of GaAs wafer
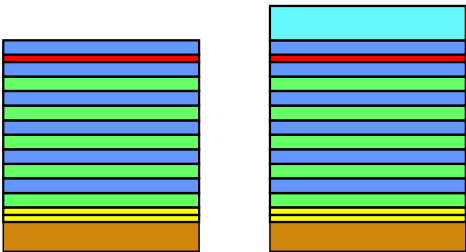

etching of etch-stop

topcoating

Using large-scale upside-down SESAMs will then be the key to limit thermal distortions of the SESAM and achieve stable modelocking in future $\mathrm{kW}$-level modelocked TDLs.

\subsection{Conclusion and Outlook}

Further average power scaling of modelocked TDLs will require larger laser spot sizes on the disk and on the SESAM. Power scaling in fundamental-mode operation with larger spot sizes on the thin disk is feasible by using state-of-the-art contacting methods and standard gain materials such as $\mathrm{Yb}$ :YAG. However, we believe these power-scaling capabilities will be extended and most likely outperformed in the near future by novel materials such as $\mathrm{Yb}: \mathrm{LuO}$, when contacting methods and growth are perfected.

Following the guidelines for high-damage threshold SESAMs presented here, using larger spot sizes on the SESAM should be straightforward. In particular, the suggested upside-down SESAMs with improved thermal management will be ideally suited for future multi-kW level intracavity average powers.

Currently, the main limitation to higher average powers is thermal effects and even thermal damage observed on intracavity components, in particular in the dispersive mirrors required to achieve stable modelocking. Designs with improved substrates and higher damage threshold material composition for better heat removal will be an important step towards reaching the next milestones in average power and pulse energy.

Given the advantages discussed in paragraph 2.1 and latest power scaling results, it seems likely that the next step in power and energy scaling of modelocked TDLs will be achieved by operating the oscillator in a vacuum environment (Figure 14). 
Figure 14. Intracavity peak power of modelocked TDLs demonstrated to date, showing the crucial advantage of operating such oscillators in a vacuum environment. The different colors indicate different gain materials that have already been modelocked in the thin-disk geometry.

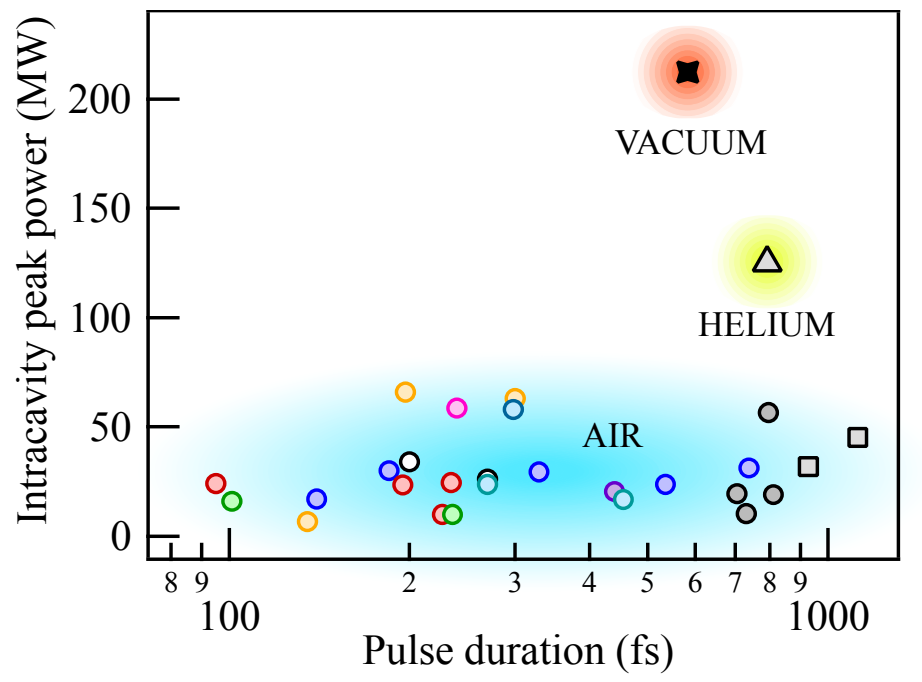

- YAG air, single pass

$\square$ YAG air, multipass

$\Delta$ YAG helium, single pass

Y YAG vacuum, single pass

- YAG Kerr-lens modelocked

- $\mathrm{LuO}$

- SSO

○ KLuW

- CALGO

○ $\mathrm{LuScO}$

- ScYLO

○ KYW

- $\mathrm{YCOB}$

With the suggested improvements, we expect further average power scaling in the near future. Furthermore, reaching pulse energies in the $100 \mu \mathrm{J}$ range already appears feasible by increasing the length of the resonator using a Herriott-type passive multi-pass cell $[32,58]$.

\section{New Pulse Duration Limits of Modelocked Thin-Disk Lasers}

Most scientific applications targeted by the development of novel high-power ultrafast sources require high peak powers in combination with short pulse durations (typically sub-100 fs), and benefit from repetition rates in the $\mathrm{MHz}$ range, resulting in increasing needs for high peak power sources with high average power. Current state-of-the-art ultrafast TDLs that combine high peak power and high average power are restricted in this aspect to pulse durations longer than $500 \mathrm{fs}$. Extending the high-power capabilities of TDLs to the sub-100 fs regime is, therefore, a major milestone, which is intimately linked to the development of novel broadband gain materials suitable for this geometry. In this paragraph, we will focus on the main challenges of combining high average power and short pulse durations, followed by a review of the latest achievements. We will mainly focus on recent experiments in which record-short pulse durations were achieved with TDLs based on the promising family of cubic sesquioxide gain materials. In particular, the recent demonstration of a modelocked TDL with sub-100-fs pulse duration based on the mixed sesquioxide material $\mathrm{Yb}$ :LuScO represents a promising first step [21]. These are the shortest pulses ever demonstrated from a TDL to date, reaching for the first time the sub-100 fs milestone. This proof-of-principle experiment shows that such oscillators are suitable to access this regime. Furthermore, preliminary power scaling experiments indicate that much higher powers are within reach. In addition, the progress in terms of pulse duration enabled the first measurement of the carrier envelope offset (CEO) frequency of a TDL, which is a first step towards full stabilization of such high-power unamplified oscillators for use in frequency comb applications. 


\subsection{Challenges}

One of the most challenging points to obtain short pulse durations and high-power levels from modelocked TDLs is finding broadband materials that combine a high mechanical strength and excellent spectroscopic properties, suitable for high-power operation and short pulse generation in the thin-disk geometry [20]. Many Yb-doped materials have been modelocked in the past years in this geometry (Figure 15). Typically, Yb-doped broadband materials exhibit a disordered lattice structure that in turn limits their thermal properties [59]. Nevertheless, the excellent heat removal capability of TDLs is suitable to overcome these limitations, and extend high-power operation to the sub-100-fs regime.

Figure 15. (a) Peak power of modelocked TDLs demonstrated to date using different gain materials versus their pulse duration; (b) Average power of modelocked TDLs demonstrated to date using different gain materials versus their pulse duration. The different results presented here and the corresponding references are listed in Table 5.

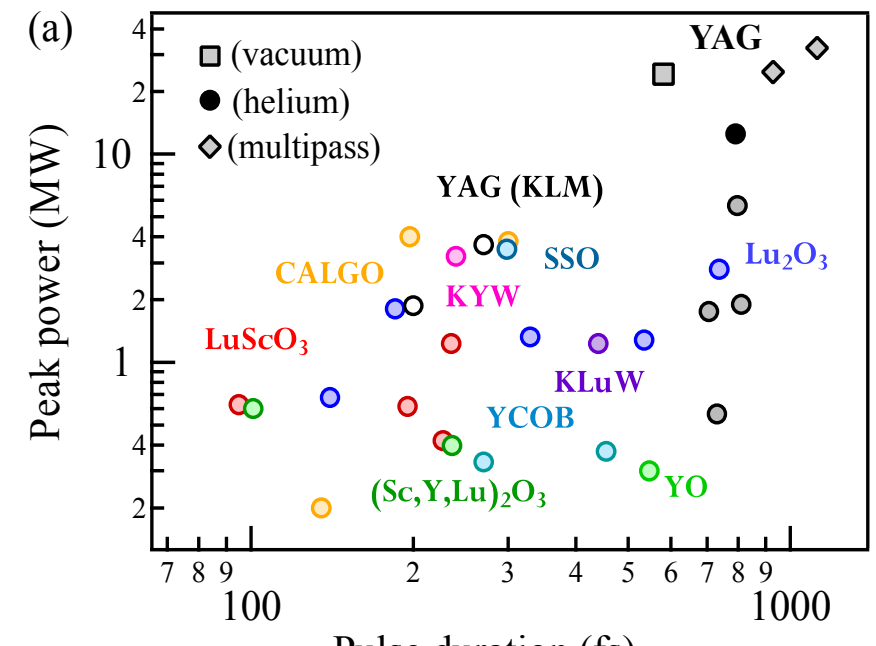

Pulse duration (fs)

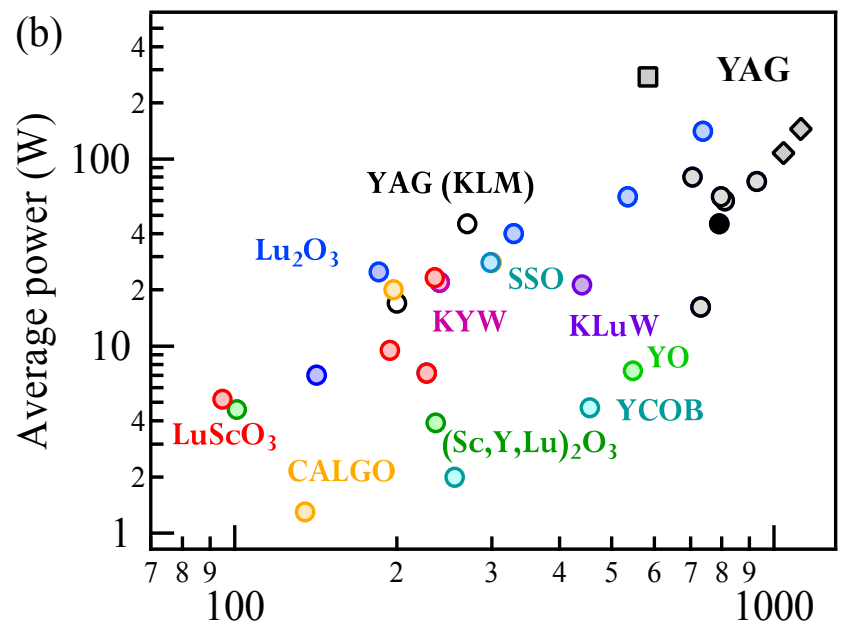

Pulse duration (fs)

Table 5. Summary of results obtained with modelocked TDLs to date. Unless otherwise indicated, the oscillators were operated in air.

\begin{tabular}{cccccccc}
\hline Material & Remarks & $\boldsymbol{P}_{\text {av }}$ & $\boldsymbol{E}_{\mathbf{p}}$ & $\boldsymbol{\tau}$ & $\boldsymbol{f}_{\text {rep }}$ & $\boldsymbol{P}$ & Ref. \\
\hline Yb:YAG & vacuum & $\mathbf{2 7 5 ~ W}$ & $17 \mu \mathrm{J}$ & $583 \mathrm{fs}$ & $16.3 \mathrm{MHz}$ & $26 \mathrm{MW}$ & {$[17]$} \\
& & $145 \mathrm{~W}$ & $\mathbf{4 1 . 3} \boldsymbol{\mu J}$ & $1.1 \mathrm{ps}$ & $3.5 \mathrm{MHz}$ & $35 \mathrm{MW}$ & {$[18]$} \\
& & $80 \mathrm{~W}$ & $1.5 \mu \mathrm{J}$ & $705 \mathrm{fs}$ & $57 \mathrm{MHz}$ & $1.7 \mathrm{MW}$ & {$[60]$} \\
& & $76 \mathrm{~W}$ & $26 \mu \mathrm{J}$ & $960 \mathrm{fs}$ & $2.9 \mathrm{MHz}$ & $25 \mathrm{MW}$ & {$[38]$} \\
& \multirow{3}{*}{ Yb:YAG } & $44 \mathrm{~W}$ & $11 \mu \mathrm{J}$ & $791 \mathrm{fs}$ & $4 \mathrm{MHz}$ & $12.5 \mathrm{MW}$ & {$[38]$} \\
& \multirow{2}{*}{ helium } & $63 \mathrm{~W}$ & $5.1 \mu \mathrm{J}$ & $796 \mathrm{fs}$ & $12 \mathrm{MHz}$ & $5.7 \mathrm{MW}$ & {$[33]$} \\
& & $60 \mathrm{~W}$ & $1.7 \mu \mathrm{J}$ & $810 \mathrm{fs}$ & $34 \mathrm{MHz}$ & $1.9 \mathrm{MW}$ & {$[44]$} \\
& & $16 \mathrm{~W}$ & $0.5 \mu \mathrm{J}$ & $730 \mathrm{fs}$ & $34 \mathrm{MHz}$ & $0.6 \mathrm{MW}$ & {$[16]$} \\
\hline
\end{tabular}


Table 5. Cont.

\begin{tabular}{|c|c|c|c|c|c|c|c|}
\hline Material & Remarks & $\boldsymbol{P}_{\mathrm{av}}$ & $E_{\mathrm{p}}$ & $\tau$ & $f_{\text {rep }}$ & $P$ & Ref. \\
\hline & Kerr Lens & & & & & & \\
\hline \multirow[t]{3}{*}{ Yb:YAG } & $\begin{array}{l}\text { Modelocking } \\
\text { (KLM) }\end{array}$ & $17 \mathrm{~W}$ & $0.4 \mu \mathrm{J}$ & $200 \mathrm{fs}$ & $40 \mathrm{MHz}$ & $1.9 \mathrm{MW}$ & [61] \\
\hline & KLM & $45 \mathrm{~W}$ & $1.1 \mu \mathrm{J}$ & $270 \mathrm{fs}$ & $40 \mathrm{MHz}$ & $3.7 \mathrm{MW}$ & [61] \\
\hline & $\begin{array}{c}\text { KLM + } \\
\text { Positive } \\
\text { dispersion } \\
\text { regime }\end{array}$ & $17 \mathrm{~W}$ & $0.4 \mu \mathrm{J}$ & $1.7 \mathrm{ps}$ & $40 \mathrm{MHz}$ & $0.2 \mathrm{MW}$ & {$[62]$} \\
\hline \multirow[t]{5}{*}{$\mathrm{Yb}: \mathrm{LuO}$} & & $141 \mathrm{~W}$ & $2.3 \mu \mathrm{J}$ & $738 \mathrm{fs}$ & $60 \mathrm{MHz}$ & $2.8 \mathrm{MW}$ & [26] \\
\hline & & $63 \mathrm{~W}$ & $0.8 \mu \mathrm{J}$ & $535 \mathrm{fs}$ & $81 \mathrm{MHz}$ & $1.3 \mathrm{MW}$ & [63] \\
\hline & & $40 \mathrm{~W}$ & $0.5 \mu \mathrm{J}$ & $329 \mathrm{fs}$ & $81 \mathrm{MHz}$ & $1.3 \mathrm{MW}$ & [63] \\
\hline & & $7 \mathrm{~W}$ & $0.1 \mu \mathrm{J}$ & $142 \mathrm{fs}$ & $64 \mathrm{MHz}$ & $0.7 \mathrm{MW}$ & [22] \\
\hline & & $25 \mathrm{~W}$ & $0.4 \mu \mathrm{J}$ & $185 \mathrm{fs}$ & $66 \mathrm{MHz}$ & $1.8 \mathrm{MW}$ & [64] \\
\hline Yb:KLuW & & $21 \mathrm{~W}$ & $0.6 \mu \mathrm{J}$ & $440 \mathrm{fs}$ & $35 \mathrm{MHz}$ & $1.2 \mathrm{MW}$ & [65] \\
\hline Yb:KYW & & $22 \mathrm{~W}$ & $0.9 \mu \mathrm{J}$ & $240 \mathrm{fs}$ & $25 \mathrm{MHz}$ & $3.3 \mathrm{MW}$ & [66] \\
\hline \multirow[t]{2}{*}{ Yb:ScYLO } & & $3.9 \mathrm{~W}$ & $0.1 \mu \mathrm{J}$ & $236 \mathrm{fs}$ & $36 \mathrm{MHz}$ & $0.4 \mathrm{MW}$ & [67] \\
\hline & & $4.6 \mathrm{~W}$ & $0.1 \mu \mathrm{J}$ & $101 \mathrm{fs}$ & $70 \mathrm{MHz}$ & $0.6 \mathrm{MW}$ & - \\
\hline Yb:SSO & & $28 \mathrm{~W}$ & $1 \mu \mathrm{J}$ & $298 \mathrm{fs}$ & $27 \mathrm{MHz}$ & $3.5 \mathrm{MW}$ & [68] \\
\hline \multirow[t]{3}{*}{ Yb:CALGO } & & $28 \mathrm{~W}$ & $1.3 \mu \mathrm{J}$ & $300 \mathrm{fs}$ & $21 \mathrm{MHz}$ & $3.8 \mathrm{MW}$ & [69] \\
\hline & & $20 \mathrm{~W}$ & $0.9 \mu \mathrm{J}$ & $197 \mathrm{fs}$ & $21 \mathrm{MHz}$ & $4 \mathrm{MW}$ & [69] \\
\hline & & $1.3 \mathrm{~W}$ & $0.03 \mu \mathrm{J}$ & $135 \mathrm{fs}$ & $45 \mathrm{MHZ}$ & $0.2 \mathrm{MW}$ & [69] \\
\hline \multirow[t]{2}{*}{ Yb:YCOB } & & $2 \mathrm{~W}$ & $0.1 \mu \mathrm{J}$ & $270 \mathrm{fs}$ & $20 \mathrm{MHz}$ & $0.3 \mathrm{MW}$ & [70] \\
\hline & & $4.7 \mathrm{~W}$ & $0.2 \mu \mathrm{J}$ & $455 \mathrm{fs}$ & $24 \mathrm{MHz}$ & $0.4 \mathrm{MW}$ & [70] \\
\hline \multirow[t]{4}{*}{ Yb:LuScO } & & $7 \mathrm{~W}$ & $0.1 \mu \mathrm{J}$ & $227 \mathrm{fs}$ & $66 \mathrm{MHz}$ & $0.4 \mathrm{MW}$ & [71] \\
\hline & & $23 \mathrm{~W}$ & $0.3 \mu \mathrm{J}$ & $235 \mathrm{fs}$ & $70 \mathrm{MHz}$ & $1.2 \mathrm{MW}$ & [72] \\
\hline & & $9.5 \mathrm{~W}$ & $0.1 \mu \mathrm{J}$ & $195 \mathrm{fs}$ & $70 \mathrm{MHz}$ & $0.6 \mathrm{MW}$ & [72] \\
\hline & & $5 \mathrm{~W}$ & $0.1 \mu \mathrm{J}$ & $96 \mathrm{fs}$ & $77 \mathrm{MHz}$ & $0.6 \mathrm{MW}$ & [21] \\
\hline
\end{tabular}

Another critical point is the maximum tolerable phase shift for soliton modelocking, which was discussed in 2.1. At a given intracavity average power, the intracavity peak power is significantly higher for shorter pulses. This sets a lower limit to the achievable average output power, which is lower than in the case of somewhat longer pulses (Figure 15, left). In addition, typical TDLs aiming for short pulse durations operate with low outcoupling rates: on the one hand because a low overall gain per roundtrip is beneficial to obtain short pulses in the soliton modelocking regime [28,29]; on the other hand, because the gain spectrum of most $\mathrm{Yb}$-doped materials usually becomes smoother at low inversion levels $\beta$ (corresponding to low output coupling rates). In Figure 16, we illustrate the effect of the inversion level on the gain spectrum in the absence of other intracavity spectrally dependent losses. As a consequence, TDLs with short pulses usually operate at a high ratio of intracavity versus output power, further limiting the achievable output peak power (Figure 15, right).

Furthermore, specific SESAM parameters are required to generate high power levels and short pulses from TDLs based on gain media with moderate gain bandwidths. The main challenges are in this case: 
- A somewhat higher modulation depth compared to what is typically used in high-power TDLs is beneficial to push the pulse duration to the bandwidth limit [28,29]. This contributes to a higher QML threshold [51]. In addition, typical gain cross-sections of broadband materials are moderate, which further contributes to a higher QML threshold. During this regime, peak powers can become significantly larger than in cw modelocked regime.

- At short pulse durations ( $<200 \mathrm{fs})$, the SESAM rollover in reflectivity can already occur at moderate pulse fluences due to TPA. As we discussed in 2.3.1, this can lead to multi-pulsing instabilities at moderate pulse energy and can be one of the main limiting factors.

- SESAMs with short recovery times are beneficial to push the pulse duration limits of such sources $[28,29]$. A common way of tailoring the recovery time of the absorber is to grow the QW absorbers at low-temperature (LT). LT-growth introduces additional defects in the lattice that result in fast nonradiative decay times [73-75]. However, this results in extra nonsaturable losses, which can lead to increased thermal effects $[52,74]$.

Although these requirements represent additional challenges, we believe that current state-of-the-art SESAMs, based on the guidelines presented in the paragraph 2.3.6 are already suitable for the next power-scaling step with sub-100 fs pulse duration.

Figure 16. Left: Gain cross section of $\mathrm{Yb}: \mathrm{LuO}$ for different inversion levels of the gain medium; Right: Gain cross section of $\mathrm{Yb}$ : $\mathrm{LuScO}$ for different inversion levels of the gain medium.

(a)

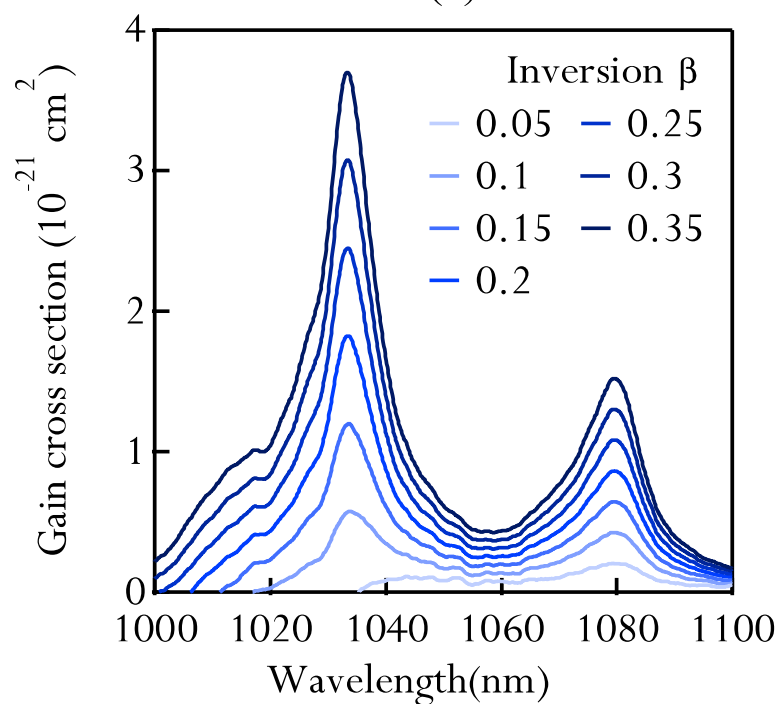

(b)

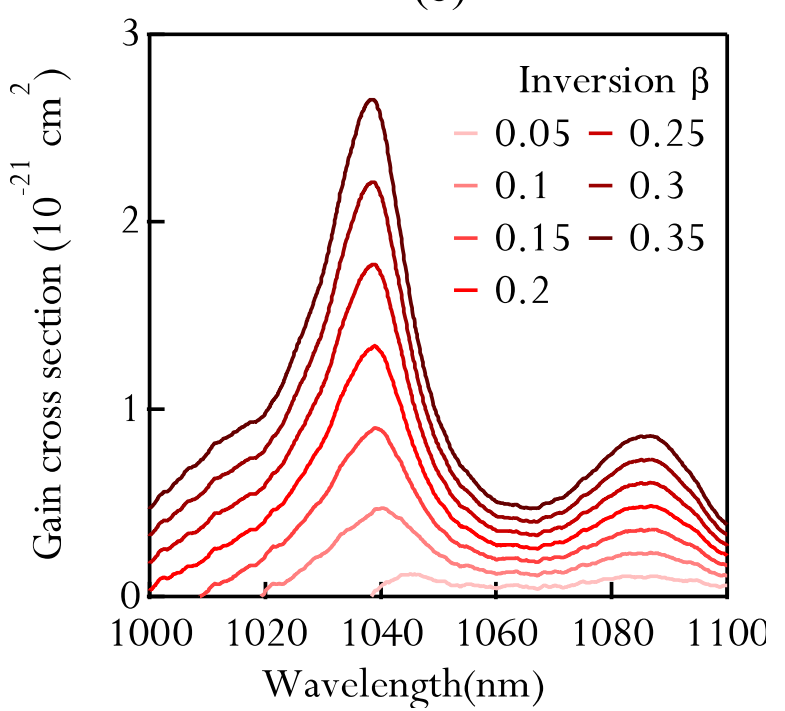

\subsection{State-of-the Art and Recent Experimental Results}

Table 5 summarizes laser results achieved to date from modelocked TDLs. These results can also be found in Figure 15. A more general overview of different Yb-doped materials for the generation of ultrashort pulses can be found in [59]. In addition, a specific overview of broadband materials for TDLs is given in reference [20].

The most widely-used gain material for TDLs is Yb:YAG [76]. It is grown with excellent quality and in large sizes. Furthermore, it has a good thermal conductivity of $\approx 6.6 \mathrm{~W} \cdot \mathrm{m}^{-1} \cdot \mathrm{K}^{-1}$ for an $\mathrm{Yb}^{3+}$ 
doping concentration of 10 at.\%, which is a key ingredient for laser operation at high power levels. The first modelocked TDL was based on Yb:YAG and delivered an average power of $16.2 \mathrm{~W}$ with a pulse duration of $730 \mathrm{fs}$ [16]. To date, the pulse duration of high-power SESAM modelocked Yb:YAG TDLs is limited to pulses longer than $500 \mathrm{fs}$, although much shorter pulses were demonstrated in low-power bulk oscillators [77-80]. Recently, the first Kerr-lens modelocked (KLM) Yb:YAG TDL was demonstrated with an average power of $17 \mathrm{~W}$ and a pulse duration of $200 \mathrm{fs}$ [61], and similar performance was obtained by operating the KLM modelocked oscillator in the positive dispersion regime [62].

One broadband material that held the record for the shortest pulses from a modelocked TDL for many years was Yb:KYW $[81,82]$. In 2002, a modelocked TDL based on this material was presented with an average power of $22 \mathrm{~W}$ and a pulse duration of $240 \mathrm{fs}$ [66]. In this result, these short pulse durations were achieved with an intracavity prism in order to tune-off the narrow maximum emission peak thus broadening and flattening the emission spectrum. The varying quality of the available material and the anisotropic nature of this crystal limited further power scaling.

Some borate materials show wide gain spectra for the generation of short pulses [83-85]. Initial modelocking experiments with $\mathrm{Yb}$ :YCOB resulted in a pulse duration of $270 \mathrm{fs}$ at a moderate average output power of $2 \mathrm{~W}$. In this case, anisotropic thermal aberrations made obtaining fundamental mode operation difficult at higher power levels [70].

The first modelocking experiments using the promising material $\mathrm{Yb}: \mathrm{CALGO}\left(\mathrm{Yb}: \mathrm{CaGdAlO}_{4}\right)[86,87]$ in the thin-disk configuration were recently reported $[69,88]$. This material combines a very broad and smooth emission bandwidth with a thermal conductivity comparable to $\mathrm{Yb}$ :YAG $\left(6.5 \mathrm{~W} \cdot \mathrm{m}^{-1} \cdot \mathrm{K}^{-1}\right.$ for $2 \%$ doping). Furthermore this material benefits from anisotropic thermal properties when cut along the $\sigma$-axis, which are crucial for power scaling in the thin-disk configuration. In the first modelocking results, up to $28 \mathrm{~W}$ of average power were demonstrated with $300 \mathrm{fs}$ pulses. Shorter pulses (135 fs) could only be achieved at a moderate average power of $1.3 \mathrm{~W}$. The available doping concentrations and thickness of the crystal limits the average power in this first experiment. Nevertheless, this material is one of the most interesting candidates for power scaling of modelocked TDLs with short pulses, in particular given the very promising results obtained with bulk modelocked lasers based on this material $[89,90]$.

Very recently, promising first results were demonstrated using the oxyorthosilicate laser crystal $\mathrm{Yb}: \mathrm{Sc}_{2} \mathrm{SiO}_{5}$ (Yb:SSO) [91,92], where $27.8 \mathrm{~W}$ and $298 \mathrm{fs}$ were demonstrated [68]. In this case, further improvements on growth and polishing quality are still required for further power scaling.

Finally, another very successful group of thin-disk gain materials are Yb-doped cubic sesquioxides. This family has been recognized as potentially suitable to outperform Yb:YAG in terms of efficiency and shorter pulse durations [93-99]. These isotropic materials combine high mechanical strength, excellent thermal properties and high absorption cross sections. Although these cubic sesquioxide materials are known since 1957 [97], the high melting temperature of about $2400{ }^{\circ} \mathrm{C}$ has limited the growth of high quality large crystals suitable TDL operation. This resulted for many years in a poor crystal quality and moderate optical-to-optical efficiencies [100,101]. In the meantime, the heat exchanger method (HEM) was developed and enabled the growth of high quality, large sesquioxide crystals suitable for the fabrication of thin-disk crystals [96]. It is worth noting that very recently, 
preliminary results were presented with a modelocked TDL based on a polycrystalline ceramic sesquioxide $\mathrm{Yb}: \mathrm{Y}_{2} \mathrm{O}_{3}$ [102].

The most promising sesquioxide material in terms of average power scaling is $\mathrm{Yb}: \mathrm{LuO}[94,103]$. This material exhibits a higher thermal conductivity than $\mathrm{Yb}: \mathrm{YAG}$ of $\kappa_{\mathrm{Lu} 3 \mathrm{O} 3}(5 \mathrm{at} . \%)=11.7 \mathrm{~W} \cdot \mathrm{m}^{-1} \cdot \mathrm{K}^{-1}$ compared to $\kappa_{\mathrm{YAG}}\left(10\right.$ at.\%) $=6.6 \mathrm{~W} \cdot \mathrm{m}^{-1} \cdot \mathrm{K}^{-1}$, for an equivalent doping concentration of $1.4 \times 10^{21} \mathrm{~cm}^{-3}$. Furthermore, it has a higher absorption cross-section at the zero-phonon line (ZPL) $(976 \mathrm{~nm})$ than Yb:YAG at its typical pump wavelength of $940 \mathrm{~nm}$. This does not only allow for fabricating thinner disks, but also reduces the thermal load in the crystal, as the quantum defect is lower. Pumping at the ZPL requires the use of volume Bragg grating stabilized diodes [104], which are currently commercially available. Pumping Yb:YAG at its ZPL (969 nm) is also possible [105]. However, in $\mathrm{Yb}: Y A G$, the peak emission cross section at the ZPL has a similar value to that of the broad peak at $940 \mathrm{~nm}$. Therefore, the benefit of pumping at the ZPL is, in this case, limited to the lower quantum defect. Given the small advantage in terms of possible efficiency and the higher constraints imposed on the pump linewidth that require using VBG stabilized diodes currently limited to $\approx 800 \mathrm{~W}$ of output power, it seems more likely that future Yb:YAG modelocked TDLs will still be pumped at its broader absorption line at $940 \mathrm{~nm}$. In addition, the availability of very thin disks with excellent quality that can be cooled efficiently further weakens the need to pump Yb:YAG at the ZPL.

In the context of modelocked TDLs, Yb:LuO exhibits nearly twice the emission bandwidth of $\mathrm{Yb}$ :YAG, making it an excellent candidate for the generation of short pulses at high power levels. The first modelocked TDL based on this material delivered an average power of $25 \mathrm{~W}$ with a pulse duration of 523 fs [103]. Subsequent power scaling led to the demonstration of an average output power of $141 \mathrm{~W}$ in $738 \mathrm{fs}$ long pulses [26], which was, for many years, the highest output power reported from a modelocked oscillator demonstrating the large potential of this material for high-power operation.

\subsubsection{Short Pulses from $\mathrm{Yb}: \mathrm{Lu}_{2} \mathrm{O}_{3}$ and First Experiment of CEO Detection of a Modelocked TDL}

The full potential of $\mathrm{Yb}: \mathrm{LuO}$ for short pulse generation in the thin-disk geometry was not exploited until the recent demonstration of $142 \mathrm{fs}$ at an average power of $7 \mathrm{~W}$ [22]. In this result, a large fraction $(\approx 70 \%)$ of the emission bandwidth of the laser material was exploited (Figure 17), confirming the potential of this material also for short pulse generation.

The short pulses of this TDL enabled the generation of a coherent super-continuum (SC), without the need for external pulse compression or amplification. The SC (Figure 18, bottom left) after the highly-nonlinear photonic crystal fiber covered more than an octave and was launched into a standard $f$-to- $2 f$ interferometer [106] for CEO beat detection. A schematic of the interferometer is shown on the top right hand side of Figure 18. The detected CEO beats had a signal-to-noise ratio (SNR) of more than $25 \mathrm{~dB}$ in a resolution bandwidth (RBW) of $3 \mathrm{kHz}$ (Figure 18, bottom right) and $30 \mathrm{~dB}$ in a RBW of $1 \mathrm{kHz}$. The achieved SNR should be large enough for initial locking tests of the CEO beat, in particular given the high stability of the observed beats. We believe better performance, in particular a higher SNR, is achievable by improving opto-mechanics, boxing, and pump operation point of the oscillator. Furthermore, optimizing the CEO-beat detection scheme (fiber length, input peak power level, fiber design, shorter pulse duration [107], temperature stabilization of the highly nonlinear fiber [108], etc.) should also result in an improved SNR. It is worth emphasizing that CEO detection 
was possible in spite of the strongly multimode pumping scheme of TDLs, usually associated with a high noise level. This seems to indicate that state-of-the-art high-power systems such as the one presented in [26] or the one presented in [17] would already be suitable to achieve 100-W-level stabilized frequency combs. Taking into account the pulse duration of such state-of-the-art systems, an external temporal pulse compression stage will be required to reach the necessary short pulse duration for CEO detection. However, we believe $100 \mathrm{~W}$ level sub-100 fs pulses will soon be obtained directly from a TDL, which will significantly simplify CEO detection.

Meanwhile, the performance of our $\mathrm{Yb}: \mathrm{LuO}$ laser with short pulse duration was improved to $25 \mathrm{~W}$ and 185 fs [64]. In this experiment, power scaling was straightforward by using larger spot sizes on the disk and on the SESAM, and by using a SESAM with a high-damage threshold, obtained following the guidelines presented in 2.3.6.

Figure 17. (a) Emission spectrum of $\mathrm{Yb}: \mathrm{LuO}$ and $\mathrm{Yb}: \mathrm{YAG}$. (b) Spectrum of the modelocked laser delivering $142 \mathrm{fs}$, showing a $8.5 \mathrm{~nm}$-wide (FWHM) modelocked spectrum, together with the $12.5 \mathrm{~nm}$ wide emission spectrum of $\mathrm{Yb}: \mathrm{LuO}$. The observed red-shift of the modelocked spectrum is due to the low inversion level at which the laser is operated. Strictly speaking, the modelocked spectrum should be compared not to the emission spectrum, but to the exact gain cross section at the inversion level $\beta$ at which the laser operates (see Figure 16). However, different sources of spectrally dependent loss (such as the reflectivity of the different dielectric mirrors in the cavity, or the SESAM) make the precise determination of $\beta$ a challenging task. Therefore, a comparison to the emission spectrum is here more adequate. (c) Autocorrelation trace of the 142 fs pulses obtained at $7 \mathrm{~W}$ of average output power.

(a)

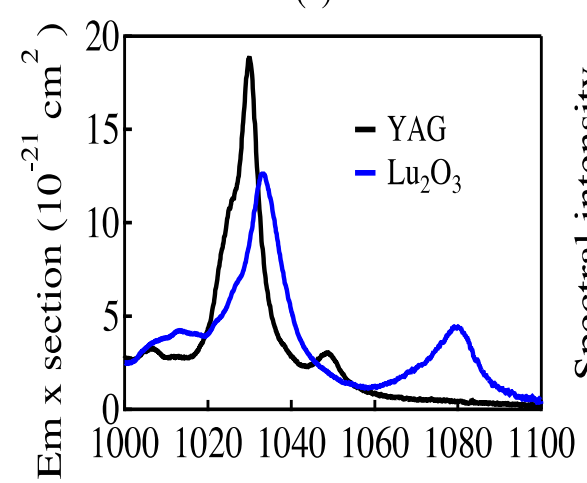

Wavelength(nm) (b)

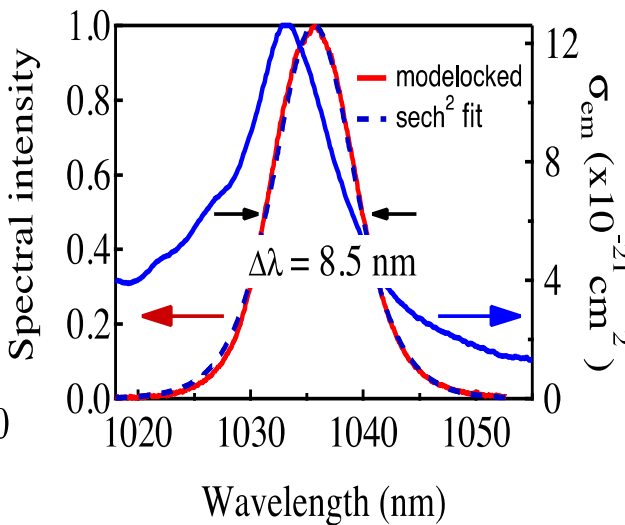

(c)

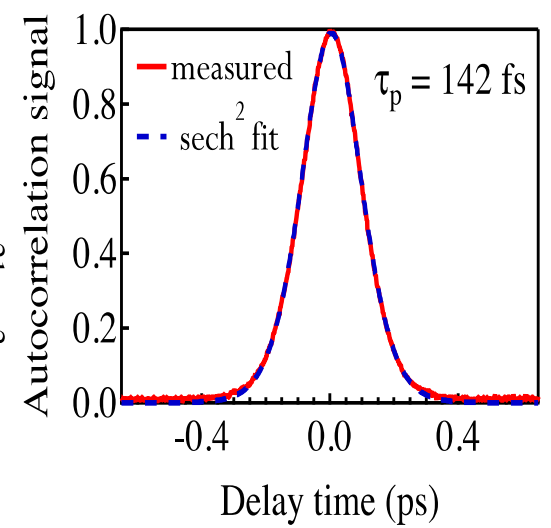


Figure 18. Setup for carrier-envelope offset (CEO) frequency detection of Yb:LuO modelocked TDL.

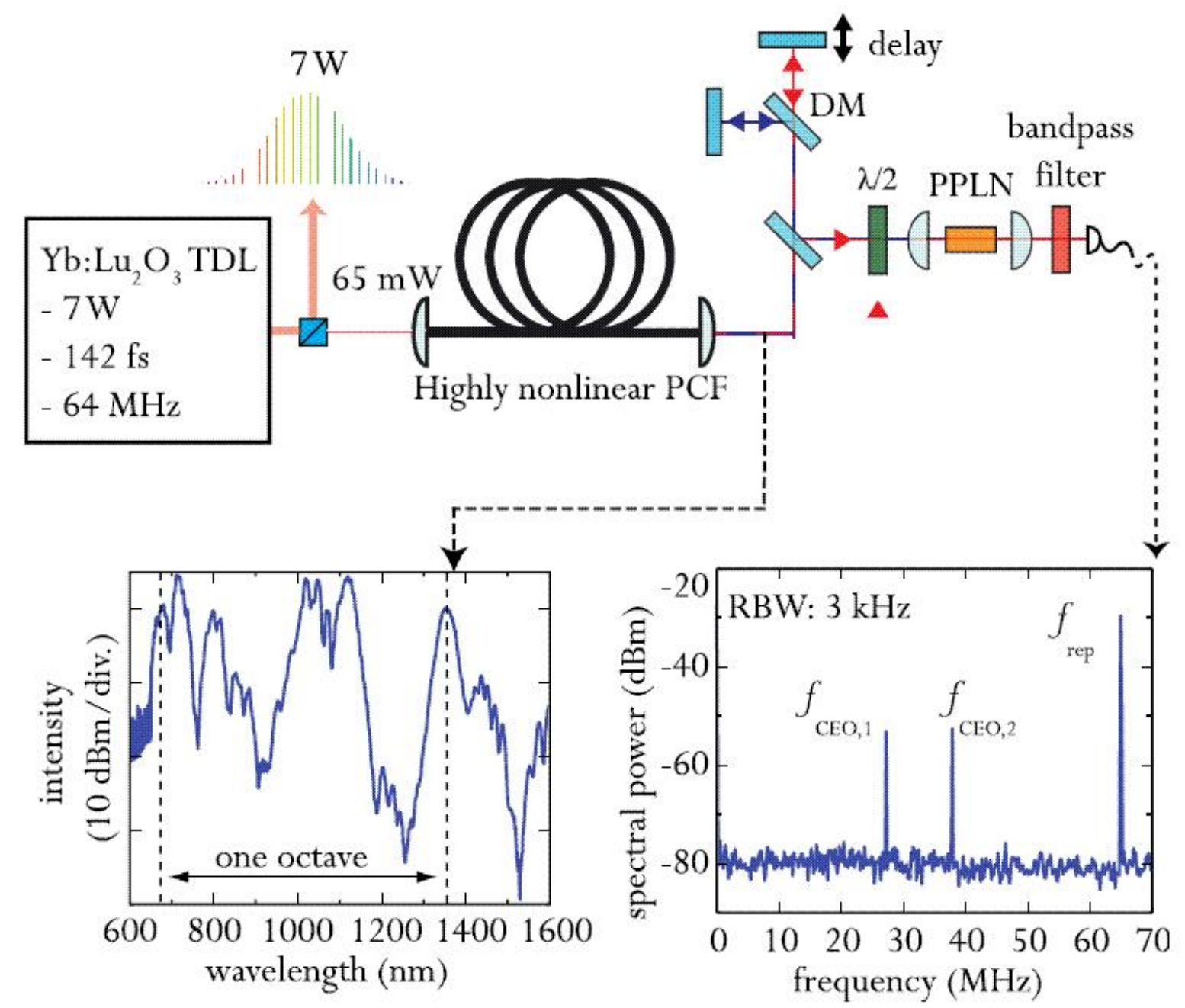

\subsubsection{Mixed Sesquioxide Materials: Sub-100 fs Pulse Duration}

The disordered mixed sesquioxide $\mathrm{Yb}: \mathrm{LuScO}$ was recently successfully introduced as an interesting candidate for short-pulse generation in the thin-disk geometry [109]. This material succeeded in combining two emission bands of $\mathrm{Yb}: \mathrm{LuO}$ and $\mathrm{Yb}: \mathrm{ScO}$ that lie roughly $7 \mathrm{~nm}$ apart, in a 22-nm-broad emission bandwidth centered around $1038 \mathrm{~nm}$. In contrast to many other broadband materials that exhibit anisotropic thermal and optical properties (such as $\mathrm{Yb}: \mathrm{KYW}$ or $\mathrm{Yb}$ :YCOB) this material has an isotropic crystal structure, which is crucial for reaching high power levels. In the first modelocking experiment, $227 \mathrm{fs}$ were reached at a moderate average power of $7.2 \mathrm{~W}$ [71]. In the meantime, the crystal quality was significantly improved and power scaling to $23 \mathrm{~W}$ with a pulse duration of $235 \mathrm{fs}$ was possible [72]. This was followed by the demonstration of a TDL based on this material with a pulse duration of $96 \mathrm{fs}$ at an average power of $5.1 \mathrm{~W}$ (Figure 19). These are the shortest pulses ever obtained from a modelocked TDL, reaching for the first time the sub-100 fs milestone [21]. The use of a fast SESAM with a relatively high modulation depth was one of the keys to exploit such a large fraction of the available bandwidth. Further SESAM optimization and the use of larger spot sizes on the disk and on the SESAM will allow for higher powers. In comparison to Yb:LuO, the larger 
emission bandwidth of $\mathrm{Yb}: \mathrm{LuScO}$ relaxes the requirements on the SESAM parameters, which should facilitate straightforward power scaling.

Another mixed sesquioxide host consisting of the mixture of $\mathrm{LuO}, \mathrm{ScO}$ and $\mathrm{YO}\left(\mathrm{Y}_{2} \mathrm{O}_{3}\right)$ was recently introduced. However, the resulting material $\mathrm{Yb}:(\mathrm{Sc}, \mathrm{Y}, \mathrm{Lu})_{2} \mathrm{O}_{3}(\mathrm{Yb}: \mathrm{ScYLO})$ showed no apparent advantage in terms of spectroscopic properties compared to $\mathrm{Yb}: \mathrm{LuScO}$. Nevertheless, it is an interesting isotropic material that exhibits an emission bandwidth of $18.5 \mathrm{~nm}$ FWHM, making it a good candidate for short pulse generation. Two thin disks from the first growth run of this material were tested in modelocked operation. In the first experiment, $236 \mathrm{fs}$ were demonstrated at an average power of $3.9 \mathrm{~W}$ [67]. The low average power was due to the very poor quality of the crystal, which showed a strongly asymmetric thermal lens and several grain boundaries, which limited the obtainable power in fundamental mode operation to only a few watts. It is worth noting that a similar problem was observed in the first results of $\mathrm{Yb}: \mathrm{LuScO}$ [71] for which the second growth run demonstrated disks of significantly higher quality.

Figure 19. (a) Emission spectrum of the mixed cubic sesquioxide $\mathrm{Yb}: \mathrm{LuScO}$ as a result of the combination of $\mathrm{Yb}: \mathrm{LuO}$ and $\mathrm{Yb}: \mathrm{ScO}$; (b) Spectrum of the modelocked laser delivering $96 \mathrm{fs}$, showing a $12.5 \mathrm{~nm}$-wide (FWHM) modelocked spectrum, together with the emission spectrum of $\mathrm{Yb}: \mathrm{LuScO}$. The observed red-shift is due to the low inversion level at which the laser operated, with an output coupling rate of 2.6\%; (c) Autocorrelation trace of the 96 fs pulses, showing clean $\operatorname{sech}^{2}$ shaped soliton pulses.

(a)

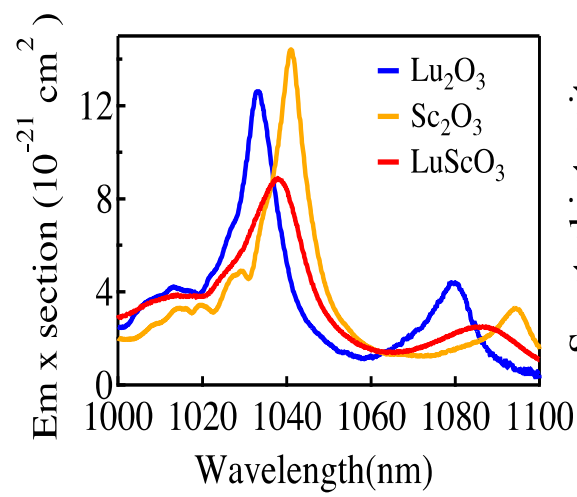

(b)

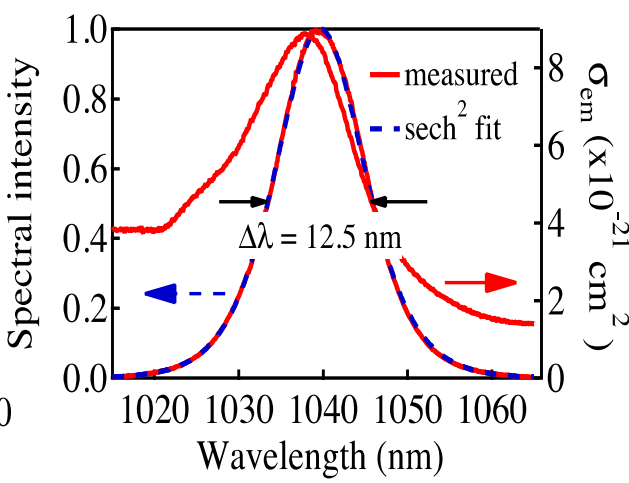

(c)

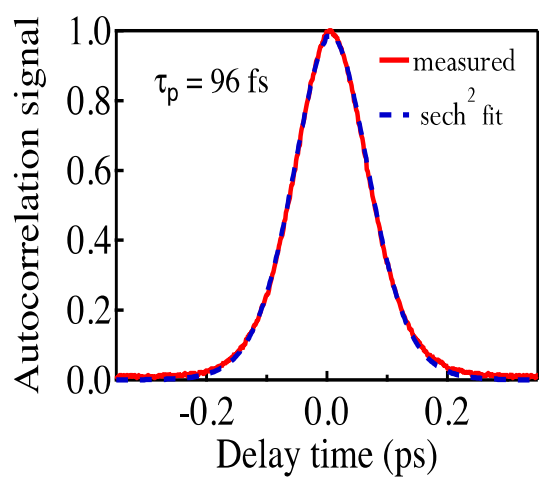

Given the quality of the available disks from the first growth run of Yb:ScYLO, we focused on exploring the limits in terms of pulse duration of this material, and were able to obtain $4.6 \mathrm{~W}$ of average power pulse duration of $101 \mathrm{fs}$. This was obtained at a repetition rate of $70 \mathrm{MHz}$, resulting in a pulse energy of $0.1 \mu \mathrm{J}$. This result further confirms the potential of this family of materials for modelocking in the thin-disk configuration with short pulse durations.

\subsection{Conclusions}

One remarkable common point of most recently achieved results is the large fraction of the available emission bandwidth that could be exploited in modelocked operation in the thin-disk geometry. This seems to indicate that much shorter pulses can be obtained with materials with significantly larger emission bandwidths, such as for example Yb:CALGO, by optimizing SESAM and soliton modelocking parameters. Furthermore, this opens the door to $100-\mathrm{W}$ level oscillators with short 
pulse durations (sub-100 fs), which would be ideally suited to drive the targeted strong-field physics experiments directly with a table-top oscillator.

Further power scaling will require thinner disks $(<100 \mu \mathrm{m})$ from improved growth runs, which should support efficient single-fundamental mode operation at high powers. The resulting low gain per single-pass will require operation in vacuum to reach the targeted $100-\mathrm{W}$ level. Furthermore, future improved dispersive mirrors and SESAM designs with low IA and high-damage thresholds such as the ones suggested in the previous paragraph will be key to support these future steps. Finally, the development of novel materials with broader emission bandwidths will be an ongoing topic of investigation.

In the meantime, although the current output power levels obtained at record-short pulse durations are still moderate, intracavity levels already reach several hundreds of watts. This already opens the door to nonlinear optics experiments driven inside a thin disk oscillator [22]. This is a promising new approach that is currently being investigated.

\section{Conclusions and Outlook}

In the last few years, the performance of modelocked TDLs was pushed to new frontiers. As a result, cutting-edge ultrafast sources were developed that will enable exciting applications, for example, in strong-field physics.

An important number of the latest achievements that were summarized in this paper were possible thanks to a thorough investigation on damage limits and crucial parameters of SESAMs for high-power oscillators. As a result of this investigation, SESAMs with ideal parameters and high-damage thresholds were achieved that enabled this technology to reach these new frontiers.

The limits in terms of average power of ultrafast oscillators were recently pushed to a new limit with the demonstration of an Yb:YAG ultrafast TDL with $275 \mathrm{~W}$ of average power. Prior to these experiments, the nonlinearity of air was recognized as one of the main contributions to the total phase shift experienced by the pulses within one round trip in the laser cavity. At high peak power, this phase shift can become too large and destabilize the modelocking mechanism. In this experiment, we reduced the influence of the nonlinearity of the ambient environment by operating the oscillator in a medium vacuum environment ( $0.5 \mathrm{mbar})$. In this way, $275 \mathrm{~W}$ of average power and a pulse energy of $16.9 \mu \mathrm{J}$ at a pulse duration of $583 \mathrm{fs}$ were achieved. In the near future, we expect this approach to enable a further increase in pulse energy and average power of such sources. Currently, the only limitations to higher average powers appear to be thermal effects that occur in the dispersive mirrors. Future thermally improved mirror designs will therefore enable the next steps in average power and pulse energy scaling.

Another important achievement was the recent demonstration of TDLs with nearly bandwidth-limited pulses that reach pulse durations as short as $96 \mathrm{fs}$. Although in these preliminary experiments we achieve moderate output powers (typically $<10 \mathrm{~W}$ ), we expect further power scaling to the $100-\mathrm{W}$ level to be within reach, in particular with tailored high-damage threshold SESAM designs, and given the latest power scaling results obtained by operating the oscillator in a vacuum environment. Furthermore, promising materials with significantly larger emission bandwidths than the ones used in this experiment should soon yield even shorter pulses at high average powers. This paves the way to 
highly nonlinear optics experiments driven directly by table-top oscillators, at high repetition rates and with high efficiency. Another promising possibility that is already enabled by the current proof-of-principle sub-100 fs medium power oscillators is to exploit the high intracavity peak powers inside TDLs to drive highly nonlinear experiments.

The record short pulses demonstrated in the context of this work enabled us to perform the first CEO frequency measurement of a modelocked TDL. The detection of this characteristic frequency is the first crucial step to fully stabilize this type of laser source, which is the key in applications such as spectroscopy or metrology.

In addition to future experiments enabled by the state-of-the-art sources presented here, the performance milestones of these modelocked thin-disk oscillators will continue to evolve to higher average power, pulse energy, peak power and shorter pulse durations.

\section{Acknowledgments}

We acknowledge financial support by the Swiss National Science Foundation (SNF) and support from the FIRST cleanroom facilities of ETH Zurich for the SESAM fabrication. Christian Kränkel and Kolja Beil acknowledge financial support by the Joachim Herz Stiftung in the framework of the Excellence Cluster "Frontiers in Quantum Photon Science" of the federal state of Hamburg. Thomas Südmeyer acknowledges support from the European Research Council for the project "Efficient megahertz XUV light source” (ERC Starting Grant 2011 \#279545).

\section{Conflict of Interest}

The authors declare no conflict of interest.

\section{References}

1. Sibbett, W.; Lagatsky, A.A.; Brown, C.T.A. The development and application of femtosecond laser systems. Opt. Express 2012, 20, 6989-7001.

2. Südmeyer, T.; Marchese, S.V.; Hashimoto, S.; Baer, C.R.E.; Gingras, G.; Witzel, B.; Keller, U. Femtosecond laser oscillators for high-field science. Nat. Photonics 2008, 2, 599-604.

3. McPherson, A.; Gibson, G.; Jara, H.; Johann, U.; Luk, T.S.; McIntyre, I.A.; Boyer, K.; Rhodes, C.K. Studies of multiphoton production of vacuum-ultraviolet radiation in the rare gases. J. Opt. Soc. Am. B 1987, 4, 595-601.

4. Ferray, M.; L’Huillier, A.; Li, X.F.; Lompré, L.A.; Mainfray, G.; Manus, C. Multiple-harmonic conversion of $1064 \mathrm{~nm}$ radiation in rare gases. J. Phys. B 1988, 21, L31-L35.

5. Keller, U. Femtosecond to attosecond optics. IEEE Photon. J. 2010, 2, 225-228.

6. Heckl, O.H.; Baer, C.R.E.; Kränkel, C.; Marchese, S.V.; Schapper, F.; Holler, M.; Südmeyer, T.; Robinson, J.S.; Tisch, J.W.G.; Couny, F.; et al. High harmonic generation in a gas-filled hollow-core photonic crystal fiber. Appl. Phys. B 2009, 97, 369-373.

7. Kim, S.; Jin, J.H.; Kim, Y.J.; Park, I.Y.; Kim, Y.; Kim, S.W. High-harmonic generation by resonant plasmon field enhancement. Nature 2008, 453, 757-760. 
8. Russbueldt, P.; Mans, T.; Weitenberg, J.; Hoffmann, H.D.; Poprawe, R. Compact diode-pumped 1.1 kW Yb:YAG Innoslab femtosecond amplifier. Opt. Lett. 2010, 34, 4169-4171.

9. Eidam, T.; Hanf, S.; Seise, E.; Andersen, T.V.; Gabler, T.; Wirth, C.; Schreiber, T.; Limpert, J.; Tünnermann, A. Femtosecond fiber CPA system emitting $830 \mathrm{~W}$ average output power. Opt. Lett. 2010, 35, 94-96.

10. Metzger, T.; Schwarz, A.; Teisset, C.Y.; Sutter, D.; Killi, A.; Kienberger, R.; Krausz, F. High-repetition-rate picosecond pump laser based on a Yb:YAG disk amplifier for optical parametric amplification. Opt. Lett. 2009, 2009, 2123-2125.

11. Giesen, A.; Hügel, H.; Voss, A.; Wittig, K.; Brauch, U.; Opower, H. Scalable concept for diodepumped high-power solid-state lasers. Appl. Phys. B 1994, 58, 365-372.

12. Giesen, A.; Speiser, J. Fifteen years of work on thin-disk lasers: results and scaling laws. IEEE J. Sel. Top. Quantum Electron. 2007, 13, 598-609.

13. Keller, U. Ultrafast solid-state laser oscillators: A success story for the last 20 years with no end in sight. Appl. Phys. B 2010, 100, 15-28.

14. Keller, U.; Weingarten, K.J.; Kärtner, F.X.; Kopf, D.; Braun, B.; Jung, I.D.; Fluck, R.; Hönninger, C.; Matuschek, N.; Aus der Au, J. Semiconductor saturable absorber mirrors (SESAMs) for femtosecond to nanosecond pulse generation in solid-state lasers. IEEE J. Sel. Top. Quantum Electron. 1996, 2, 435-453.

15. Keller, U.; Miller, D.A.B.; Boyd, G.D.; Chiu, T.H.; Ferguson, J.F.; Asom, M.T. Solid-state low-loss intracavity saturable absorber for Nd:YLF lasers: An antiresonant semiconductor Fabry-Perot saturable absorber. Opt. Lett. 1992, 17, 505-507.

16. Aus der Au, J.; Spühler, G.J.; Südmeyer, T.; Paschotta, R.; Hövel, R.; Moser, M.; Erhard, S.; Karszewski, M.; Giesen, A.; Keller, U. $16.2 \mathrm{~W}$ average power from a diode-pumped femtosecond Yb:YAG thin disk laser. Opt. Lett. 2000, 25, 859-861.

17. Saraceno, C.J.; Emaury, F.; Heckl, O.H.; Baer, C.R.E.; Hoffmann, M.; Schriber, C.; Golling, M.; Sudmeyer, T.; Keller, U. $275 \mathrm{~W}$ average output power from a femtosecond thin disk oscillator operated in a vacuum environment. Opt. Express 2012, 20, 23535-23541.

18. Bauer, D.; Zawischa, I.; Sutter, D.H.; Killi, A.; Dekorsy, T. Mode-locked Yb:YAG thin-disk oscillator with $41 \mu \mathrm{J}$ pulse energy at $145 \mathrm{~W}$ average infrared power and high power frequency conversion. Opt. Express 2012, 20, 9698-9704.

19. Saraceno, C.J.; Schriber, C.; Mangold, M.; Hoffmann, M.; Heckl, O.H.; Baer, C.R.E.; Golling, M.; Südmeyer, T.; Keller, U. SESAMs for high-power oscillators: Design guidelines and damage thresholds. IEEE J. Sel. Top. Quantum Electron. 2012, 18, $29-41$.

20. Südmeyer, T.; Kränkel, C.; Baer, C.R.E.; Heckl, O.H.; Saraceno, C.J.; Golling, M.; Peters, R.; Petermann, K.; Huber, G.; Keller, U. High-power ultrafast thin disk laser oscillators and their potential for sub-100-femtosecond pulse generation. Appl. Phys. B 2009, 97, 281-295.

21. Saraceno, C.J.; Heckl, O.H.; Baer, C.R.E.; Schriber, C.; Golling, M.; Beil, K.; Kränkel, C.; Südmeyer, T.; Huber, G.; Keller, U. Sub-100 femtosecond pulses from a SESAM modelocked thin disk laser. Appl. Phys. B 2012, 106, 559-562.

22. Saraceno, C.J.; Pekarek, S.; Heckl, O.H.; Baer, C.R.E.; Schriber, C.; Golling, M.; Beil, K.; Kränkel, C.; Huber, G.; Keller, U.; et al. Self-referenceable frequency comb from an ultrafast thin disk laser. Opt. Express 2012, 20, 9650-9656. 
23. Huonker, M.; Voss, A.; Schmitz, C. Laserverstärkersystem: Offenlegungsschrift des deutschen Patent-und Markenamts (DE 10061424 A 1), 2000.

24. Mende, J.; Schmid, E.; Speiser, J.; Spindler, G.; Giesen, A. Thin disk laser: Power scaling to the $\mathrm{kW}$ regime in fundamental mode operation. Proc. SPIE 2009, 7193, doi:10.1117/12.809031.

25. Killi, A.; Stolzenburg, C.; Zawischa, I.; Sutter, D.; Kleinbauer, J.; Schad, S.; Brockmann, R.; Weiler, S.; Neuhaus, J.; Kalfhues, S.; et al. The broad applicability of the disk laser principle - from CW to ps. Proc. SPIE 2009, 7193, doi:10.1117/12.808255.

26. Baer, C.R.E.; Kränkel, C.; Saraceno, C.J.; Heckl, O.H.; Golling, M.; Peters, R.; Petermann, K.; Südmeyer, T.; Huber, G.; Keller, U. Femtosecond thin disk laser with $141 \mathrm{~W}$ of average power. Opt. Lett. 2010, 35, 2302-2304.

27. Baer, C.R.E.; Heck1, O.H.; Saraceno, C.J.; Schriber, C.; Kränkel, C.; Südmeyer, T.; Keller, U. Frontiers in passively mode-locked high-power thin disk laser oscillators. Opt. Express 2012, 20, 7054-7065.

28. Kärtner, F.X.; Keller, U. Stabilization of soliton-like pulses with a slow saturable absorber. Opt. Lett. 1995, 20, 16-18.

29. Paschotta, R.; Keller, U. Passive mode locking with slow saturable absorbers. Appl. Phys. B 2001, 73, 653-662.

30. Kuhl, J.; Heppner, J. Compression of femtosecond optical pulses with dielctric multilayer interferometers. IEEE Trans. Quantum Electron. 1986, QE-22, 182-185.

31. Heppner, J.; Kuhl, J. Intracavity chirp compensation in a colliding pulse mode-locked laser using thin-film interferometers. Appl. Phys. Lett. 1985, 47, 453-456.

32. Marchese, S.V.; Baer, C.R.E.; Engqvist, A.G.; Hashimoto, S.; Maas, D.J.H.C.; Golling, M.; Südmeyer, T.; Keller, U. Femtosecond thin disk laser oscillator with pulse energy beyond the 10-microjoule level. Opt. Express 2008, 16, 6397-6407.

33. Marchese, S.V.; Südmeyer, T.; Golling, M.; Grange, R.; Keller, U. Pulse energy scaling to $5 \mu \mathrm{J}$ from a femtosecond thin disk laser. Opt. Lett. 2006, 31, 2728-2730.

34. Paschotta, R.; Häring, R.; Keller, U.; Garnache, A.; Hoogland, S.; Tropper, A.C. Soliton-like pulse-shaping mechanism in passively mode-locked surface-emitting semiconductor lasers. Appl. Phys. B 2002, 75, 445-451.

35. Nibbering, E.T.J.; Grillon, G.; Franco, M.A.; Prade, B.S.; Mysyrowicz, A. Determination of the inertial contribution to the nonlinear refractive index of air, $\mathrm{N}_{2}$, and $\mathrm{O}_{2}$ by use of unfocused high-intensity femtosecond laser pulses. J. Opt. Soc. Am. B 1997, 14, 650-660.

36. Lehmeier, H.J.; Leupacher, W.; Penzkofer, A. Nonresonant 3rd order hyperpolarizability of raregases and $\mathrm{n} 2$ determined by 3rd harmonic-generation. Opt. Commun. 1985, 56, 67-72.

37. Neuhaus, J.; Kleinbauer, J.; Killi, A.; Weiler, S.; Sutter, D.; Dekorsy, T. Passively mode-locked $\mathrm{Yb}$ :YAG thin-disk laser with pulse energies exceeding $13 \mu \mathrm{J}$ by use of an active multipass geometry. Opt. Lett. 2008, 33, 726-728.

38. Neuhaus, J.; Bauer, D.; Zhang, J.; Killi, A.; Kleinbauer, J.; Kumkar, M.; Weiler, S.; Guina, M.; Sutter, D.H.; Dekorsy, T. Subpicosecond thin-disk laser oscillator with pulse energies of up to 25.9 microjoules by use of an active multipass geometry. Opt. Express 2008, 16, 20530-20539.

39. Shimoji, Y.; Fay, A.T.; Chang, R.S.F.; Djeu, N. Direct measurement of the nonlinear refractiveindex of air. J. Opt. Soc. Am. B 1989, 6, 1994-1998. 
40. Spühler, G.J.; Weingarten, K.J.; Grange, R.; Krainer, L.; Haiml, M.; Liverini, V.; Golling, M.; Schon, S.; Keller, U. Semiconductor saturable absorber mirror structures with low saturation fluence. Appl. Phys. B 2005, 81, 27-32.

41. Maas, D.J.H.C.; Bellancourt, A.R.; Hoffmann, M.; Rudin, B.; Barbarin, Y.; Golling, M.; Südmeyer, T.; Keller, U. Growth parameter optimization for fast quantum dot SESAMs. Opt. Express 2008, 16, 18646-18656.

42. Schättiger, F.; Bauer, D.; Demsar, J.; Dekorsy, T.; Kleinbauer, J.; Sutter, D.H.; Puustinen, J.; Guina, M. Characterization of InGaAs and InGaAsN semiconductor saturable absorber mirrors for high-power mode-locked thin-disk lasers. Appl. Phys. B 2011, 106, 605-612.

43. Schibli, T.R.; Thoen, E.R.; Kärtner, F.X.; Ippen, E.P. Suppression of Q-switched mode locking and break-up into multiple pulses by inverse saturable absorption. Appl. Phys. B 2000, 70, S41-S49.

44. Innerhofer, E.; Südmeyer, T.; Brunner, F.; Häring, R.; Aschwanden, A.; Paschotta, R.; Keller, U.; Hönninger, C.; Kumkar, M. $60 \mathrm{~W}$ average power in 810-fs pulses from a thin-disk Yb:YAG laser. Opt. Lett. 2003, 28, 367-369.

45. Grange, R.; Haiml, M.; Paschotta, R.; Spuhler, G.J.; Krainer, L.; Golling, M.; Ostinelli, O.; Keller, U. New regime of inverse saturable absorption for self-stabilizing passively mode-locked lasers. Appl. Phys. B 2005, 80, 151-158.

46. Maas, D.J.H.C.; Rudin, B.; Bellancourt, A.-R.; Iwaniuk, D.; Marchese, S.V.; Südmeyer, T.; Keller, U. High precision optical characterization of semiconductor saturable absorber mirrors. Opt. Express 2008, 16, 7571-7579.

47. Haiml, M.; Grange, R.; Keller, U. Optical characterization of semiconductor saturable absorbers. Appl. Phys. B 2004, 79, 331-339.

48. Thoen, E.R.; Koontz, E.M.; Joschko, M.; Langlois, P.; Schibli, T.R.; Kärtner, F.X.; Ippen, E.P.; Kolodziejski, L.A. Two-photon absorption in semiconductor saturable absorber mirrors. Appl. Phys. Lett. 1999, 74, 3927-3929.

49. Maas, D.J.H.C. MIXSELs-A New Class of Ultrafast Semiconductor Lasers. In Proceeding of Lasers and Electro-Optics, 2007 and the International Quantum Electronics Conference, Munich, Germany, 17-22 June 2007.

50. Van Stryland, E.W.; Woodall, M.A.; Vanherzeele, H.; Soileau, M.J. Energy band-gap dependence of two-photon absorption. Opt. Lett. 1985, 10, 490-492.

51. Hönninger, C.; Paschotta, R.; Morier-Genoud, F.; Moser, M.; Keller, U. Q-switching stability limits of continuous-wave passive mode locking. J. Opt. Soc. Am. B 1999, 16, 46-56.

52. Haiml, M.; Siegner, U.; Morier-Genoud, F.; Keller, U.; Luysberg, M.; Lutz, R.C.; Specht, P.; Weber, E.R. Optical nonlinearity in low-temperature-grown GaAs: Microscopic limitations and optimization strategies. Appl. Phys. Lett. 1999, 74, 3134-3136.

53. Schieffer, S.L.; Berger, J.A.; Rickman, B.L.; Nayyar, V.P.; Schroeder, W.A. Thermal effects in semiconductor saturable-absorber mirrors. J. Opt. Soc. Am. B 2012, 29, 543-552.

54. Keller, U.; Tropper, A.C. Passively modelocked surface-emitting semiconductor lasers. Phys. Rep. 2006, 429, 67-120.

55. Kuznetsov, M.; Hakimi, F.; Sprague, R.; Mooradian, A. Design and characteristics of high-power $(>0.5-\mathrm{w} \mathrm{cw})$ diode-pumped vertical-external-cavity surface-emitting semiconductor lasers with circular tem 00 beams. IEEE J. Sel. Top. Quantum Electron. 1999, 5, 561-573. 
56. Chilla, J.; Butterworth, S.; Zeitschel, A.; Charles, J.; Caprara, A.; Reed, M.; Spinelli, L. High power optically pumped semiconductor lasers. Proc. SPIE 2004, doi:10.1117/12.549003.

57. Südmeyer, T.; Maas, D.J.H.C.; Keller, U. Mode-locked Semiconductor Disk Lasers. In Semiconductor Disk Lasers; Okhotnikov, O., Ed.; Wiley-VCH Verlag GmbH \& Co. KGaA: Weinheim, Germany, 2010.

58. Herriott, D.; Kogelnik, H.; Kompfner, R. Off-axis paths in spherical mirror interferometers. Appl. Opt. 1964, 3, 523-526.

59. Druon, F.; Balembois, F.; Georges, P. Laser crystals for the production of ultra-short laser pulses. Ann. Chim. Sci. Mat. 2003, 28, 47-72.

60. Brunner, F.; Innerhofer, E.; Marchese, S.V.; Südmeyer, T.; Paschotta, R.; Usami, T.; Ito, H.; Kurimura, S.; Kitamura, K.; Arisholm, G.; et al. Powerful red-green-blue laser source pumped with a mode-locked thin disk laser. Opt. Lett. 2004, 29, 1921-1923.

61. Pronin, O.; Brons, J.; Grasse, C.; Pervak, V.; Boehm, G.; Amann, M.C.; Kalashnikov, V.L.; Apolonski, A.; Krausz, F. High-power 200 fs Kerr-lens mode-locked Yb:YAG thin-disk oscillator. Opt. Lett. 2011, 36, 4746-4748.

62. Pronin, O.; Brons, J.; Grasse, C.; Pervak, V.; Boehm, G.; Amann, M.C.; Apolonski, A.; Kalashnikov, V.L.; Krausz, F. High-power Kerr-lens mode-locked Yb:YAG thin-disk oscillator in the positive dispersion regime. Opt. Lett. 2012, 37, 3543-3545.

63. Baer, C.R.E.; Kränkel, C.; Saraceno, C.J.; Heckl, O.H.; Golling, M.; Südmeyer, T.; Peters, R.; Petermann, K.; Huber, G.; Keller, U. Femtosecond $\mathrm{Yb}: \mathrm{Lu}_{2} \mathrm{O}_{3}$ thin disk laser with $63 \mathrm{~W}$ of average power. Opt. Lett. 2009, 34, 2823-2825.

64. Saraceno, C.J.; Schriber, C.; Heckl, O.H.; Baer, C.R.E.; Golling, M.; Beil, K.; Kränkel, C.; Südmeyer, T.; Huber, G.; Keller, U. 25 W, 185 fs Pulses from an Yb:Lu2O3 Modelocked Thin Disk Laser. In Proceedings of Europhoton 2012-5th EPS-QEOD EUROPHOTON CONFERENCE, Stockholm, Sweden, 26-31 August 2012.

65. Palmer, G.; Schultze, M.; Siegel, M.; Emons, M.; Bünting, U.; Morgner, U. Passively mode-locked $\mathrm{Yb}: \mathrm{KLu}\left(\mathrm{WO}_{4}\right)_{2}$ thin-disk oscillator operated in the positive and negative dispersion regime. Opt. Lett. 2008, 33, 1608-1610.

66. Brunner, F.; Südmeyer, T.; Innerhofer, E.; Paschotta, R.; Morier-Genoud, F.; Gao, J.; Contag, K.; Giesen, A.; Kisel, V.E.; Shcherbitsky, V.G.; et al. 240-fs pulses with 22-W average power from a mode-locked thin-disk $\mathrm{Yb}: \mathrm{KY}\left(\mathrm{WO}_{4}\right)_{2}$ laser. Opt. Lett. 2002, 27, 1162-1164.

67. Saraceno, C.J.; Heckl, O.H.; Baer, C.R.E.; Golling, M.; Südmeyer, T.; Beil, K.; Kränkel, C.; Petermann, K.; Huber, G.; Keller, U. CW and Modelocked Operation of an Yb:(Sc,Y,Lu)(2)O(3) Thin-disk Laser. In Proceedings of 2011 Conference on Lasers and Electro-Optics (CLEO), Munich, Germany, 4 May 2011.

68. Wentsch, K.; Zheng, L.; Xu, J.; Abdou-Ahmed, M.; Graf, T. Passively mode-locked $\mathrm{Yb} 3+: \mathrm{Sc}_{2} \mathrm{SiO}_{5}$ thin-disk laser. Opt. Lett. 2012, 37, 4750-4753.

69. Ricaud, S.; Jaffres, A.; Wentsch, K.; Suganuma, A.; Viana, B.; Loiseau, P.; Weichelt, B.; Abdou-Ahmed, M.; Voss, A.; Graf, T.; et al. Femtosecond Yb:CaGdAlO4 thin-disk oscillator. Opt. Lett. 2012, 37, 3984-3987. 
70. Heckl, O.H.; Kränkel, C.; Baer, C.R.E.; Saraceno, C.J.; Südmeyer, T.; Petermann, K.; Huber, G.; Keller, U. Continuous-wave and modelocked Yb:YCOB thin disk laser: First demonstration and future prospects. Opt. Express 2010, 18, 19201-19208.

71. Baer, C.R.E.; Kränkel, C.; Heckl, O.H.; Golling, M.; Südmeyer, T.; Peters, R.; Petermann, K.; Huber, G.; Keller, U. 227-fs pulses from a mode-locked yb:lusco 3 thin disk Laser. Opt. Express 2009, 17, 10725-10730.

72. Saraceno, C.J.; Heckl, O.H.; Baer, C.R.E.; Golling, M.; Südmeyer, T.; Beil, K.; Kränkel, C.; Petermann, K.; Huber, G.; Keller, U. SESAMs for high-power femtosecond modelocking: Power scaling of an $\mathrm{Yb}: \mathrm{LuScO} 3$ thin disk laser to $23 \mathrm{~W}$ and 235 fs. Opt. Express 2011, 19, 20288-20300.

73. Lochtefeld, A.J.; Melloch, M.R.; Chang, J.C.P.; Harmon, E.S. The role of point defects and arsenic precipitates in carrier trapping and recombination in low-temperature grown GaAs. Appl. Phys. Lett. 1996, 69, 1465-1467.

74. Siegner, U.; Fluck, R.; Zhang, G.; Keller, U. Ultrafast high-intensity nonlinear absorption dynamics in low-temperature grown gallium arsenide. Appl. Phys. Lett. 1996, 69, 2566-2568.

75. Loka, H.S.; Benjamin, S.D.; Smith, P.W.E. Optical Characterization of low-temperature-grown gaas for ultrafast all-optical switching devices. IEEE J. Quantum Electron. 1998, 34, 1426-1437.

76. Lacovara, P.; Choi, H.K.; Wang, C.A.; Aggarwal, R.L.; Fan, T.Y. Room-temperature diode-pumped Yb:YAG laser. Opt. Lett. 1991, 16, 1089-1091.

77. Hönninger, C.; Paschotta, R.; Graf, M.; Morier-Genoud, F.; Zhang, G.; Moser, M.; Biswal, S.; Nees, J.; Braun, A.; Mourou, G.A.; et al. Ultrafast ytterbium-doped bulk lasers and laser amplifiers. Appl. Phys. B 1999, 69, 3-17.

78. Hönninger, C.; Zhang, G.; Keller, U.; Giesen, A. Femtosecond Yb:YAG laser using semiconductor saturable absorbers. Opt. Lett. 1995, 20, 2402-2404.

79. Uemura, S.; Torizuka, K. Kerr-lens mode-locked diode-pumped Yb:YAG laser with the transverse mode passively stabilized. Appl. Physics Express 2008, 1, doi:10.1143/APEX.1.012007.

80. Uemura, S.; Torizuka, K. Sub-40-fs pulses from a diode-pumped kerr-lens mode-locked yb-doped yttrium aluminum garnet laser. Jpn. J. Appl. Phys. 2011, 50, 1-3.

81. Kuleshov, N.V.; Lagatsky, A.A.; Shcherbitsky, V.G.; Mikhailov, V.P.; Heumann, E.; Jensen, T.; Diening, A.; Huber, G. CW laser performance of $\mathrm{Yb}$ and Er,Yb doped tungstates. Appl. Phys. B 1997, 64, 409-413.

82. Kuleshov, N.V.; Lagatsky, A.A.; Podlipensky, A.V.; Mikhailov, V.P.; Huber, G. Pulsed laser operation of $\mathrm{Yb}$-doped $\mathrm{KY}\left(\mathrm{WO}_{4}\right)_{2}$ and $\mathrm{KGd}\left(\mathrm{WO}_{4}\right)_{2}$. Opt. Lett. 1997, 22, 1317-1319.

83. Kränkel, C.; Johannsen, J.; Peters, R.; Petermann, K.; Huber, G. Continuous-wave high power laser operation and tunability of $\mathrm{Yb}: \mathrm{LaSc}_{3}\left(\mathrm{BO}_{3}\right)_{4}$ in thin disk configuration. Appl. Phys. B 2007, 87, 217-220.

84. Kränkel, C.; Peters, R.; Petermann, K.; Loiseau, P.; Aka, G.; Huber, G. Efficient continuous-wave thin disk laser operation of $\mathrm{Yb}: \mathrm{Ca}_{4} \mathrm{YO}\left(\mathrm{BO}_{3}\right)_{3}$ in EIIZ and EIIX orientations with 26 W output power. J. Opt. Soc. Am. B 2009, 26, 1310-1314.

85. Valentine, G.J.; Kemp, A.J.; Birkin, D.J.L.; Burns, D.; Balembois, F.; Georges, P.; Bernas, H.; Aron, A.; Aka, G.; Sibbett, W.; et al. Femtosecond Yb:YCOB laser pumped by narrow-stripe laser diode and passively modelocked using ion implanted saturable-absorber mirror. Electron. Lett. 2000, 36, 1621-1623. 
86. Zaouter, Y.; Didierjean, J.; Balembois, F.; Lucas Leclin, G.; Druon, F.; Georges, P.; Petit, J.; Goldner, P.; Viana, B. 47-fs diode-pumped $\mathrm{Yb}^{3+}: \mathrm{CaGdAlO}_{4}$ laser. Opt. Lett. 2006, 31, 119-121.

87. Gomes, L.A.; Orsila, L.; Jouhti, T.; Okhotnikov, O.G. Picosecond SESAM-based ytterbium mode-locked fiber lasers. IEEE J. Sel. Top. Quant. 2004, 10, 129-136.

88. Ricaud, S.; Jaffres, A.; Loiseau, P.; Viana, B.; Weichelt, B.; Abdou-Ahmed, M.; Voss, A.; Graf, T.; Rytz, D.; Delaigue, M.; et al. Yb:CaGdAlO4 thin-disk laser. Opt. Lett. 2011, 36, 4134-4137.

89. Agnesi, A.; Greborio, A.; Pirzio, F.; Reali, G.; Au, J.A.D.; Guandalini, A. 40-fs Yb3+: CaGdAlO4 laser pumped by a single-mode 350-mW laser diode. Opt. Express 2012, 20, 10077-10082.

90. Greborio, A.; Guandalini, A.; Au, J.A.D. Sub-100 fs pulses with 12.5-W from Yb:CALGO based oscillators. Proc. SPIE 2012, 8235, doi:10.1117/12.906575.

91. Li, J.F.; Liang, X.Y.; He, J.P.; Zheng, L.H.; Zhao, Z.W.; Xu, J. Diode pumped passively mode-locked Yb:SSO laser with 2. 3ps duration. Opt. Express 2010, 18, 18354-18359.

92. Wentsch, K.S.; Weichelt, B.; Zheng, L.H.; Xu, J.; Ahmed, M.A.; Graf, T. Continuous-wave $\mathrm{Yb}$-doped $\mathrm{Sc}_{2} \mathrm{SiO}_{5}$ thin-disk laser. Opt. Lett. 2012, 37, 37-39.

93. Liu, J.; Rico, M.; Griebner, U.; Petrov, V.; Peters, V.; Petermann, K.; Huber, G. Efficient room temperature continuous-wave operation of an $\mathrm{Yb}^{3+}: \mathrm{Sc}_{2} \mathrm{O}_{3}$ crystal laser at 1041.6 and $1094.6 \mathrm{~nm}$. Phys. Stat. Sol. 2005, 202, R19-R21.

94. Peters, R.; Kränkel, C.; Petermann, K.; Huber, G. Broadly tunable high-power $\mathrm{Yb}: \mathrm{Lu}_{2} \mathrm{O}_{3}$ thin disk laser with 80\% slope efficiency. Opt. Express 2007, 15, 7075-7082.

95. Fornasiero, L.; Mix, E.; Peters, V.; Petermann, K.; Huber, G. New oxide crystals for solid state lasers. Cryst. Res. Technol. 1999, 34, 255-260.

96. Peters, R.; Kränkel, C.; Petermann, K.; Huber, G. Crystal growth by the heat exchanger method, spectroscopic characterization and laser operation of high-purity $\mathrm{Yb}: \mathrm{Lu}_{2} \mathrm{O}_{3}$. J. Cryst. Growth 2008, 310, 1934-1938.

97. Barta, C.; Petru, F.; Hajek, B. Über die Darstellung des Einkristalls von Scandiumoxid. Naturwissenschaften 1958, 45, 36-39.

98. Petermann, K.; Fornasiero, L.; Mix, E.; Peters, V. High melting sesquioxides: Crystal growth, spectroscopy, and laser experiments. Opt. Mater. 2002, 19, 67-71.

99. Petermann, K.; Huber, G.; Fornasiero, L.; Kuch, S.; Mix, E.; Peters, V.; Basun, S.A. Rare-earth-doped sesquioxides. J. Lumin. 2000, 87-89, 973-975.

100. Petermann, K.; Fagundes-Peters, D.; Johannsen, J.; Mond, M.; Peters, V.; Romero, J.J.; Kutovoi, S.; Speiser, J.; Giesen, A. Highly Yb-doped oxides for thin-disc lasers. J. Crys. Growth 2005, 275, 135-140.

101. Klopp, P.; Petrov, V.; Griebner, U.; Petermann, K.; Peters, V.; Erbert, G. Highly efficient mode-locked $\mathrm{Yb}: \mathrm{Sc}_{2} \mathrm{O}_{3}$ laser. Opt. Lett. 2004, 29, 391-393.

102. Tokurakawa, M.; Shirakawa, A.; Ueda, K.; Yagi, H.; Yanagitani, T.; Kaminskii, A.A.; Beil, K.; Kränkel, C.; Huber, G. Continous wave and mode-locked Yb3+:Y2O3 ceramic thin-disk laser. Opt. Express 2012, 20, 10847-10852.

103. Marchese, S.V.; Baer, C.R.E.; Peters, R.; Kränkel, C.; Engqvist, A.G.; Golling, M.; Maas, D.J.H.C.; Petermann, K.; Südmeyer, T.; Huber, G.; et al. Efficient femtosecond high power $\mathrm{Yb}: \mathrm{Lu}_{2} \mathrm{O}_{3}$ thin disk laser. Opt. Express 2007, 15, 16966-16971. 
104. Venus, G.B.; Sevian, A.; Smirnov, V.I.; Glebov, L.B. High-Brightness Narrow-Line Laser Diode Source with Volume Bragg-Grating Feedback. Proc. SPIE 2005, 5711, doi:10.1117/12.851382.

105. Weichelt, B.; Voss, A.; Ahmed, M.A.; Graf, T. Enhanced performance of thin-disk lasers by pumping into the zero-phonon line. Opt.Lett. 2012, 37, 3045-3047.

106. Telle, H.R.; Steinmeyer, G.; Dunlop, A.E.; Stenger, J.; Sutter, D.H.; Keller, U. Carrier-envelope offset phase control: A novel concept for absolute optical frequency measurement and ultrashort pulse generation. Appl. Phys. B 1999, 69, 327-332.

107. Pekarek, S.; Südmeyer, T.; Lecomte, S.; Stefan, K.; Dudley, J.M.; Keller, U. Self-referencable frequency comb from a gigahertz diode-pumped solid state laser. Opt. Express 2011, 19, $16491-16497$.

108. Heinecke, D.C.; Bartels, A.; Diddams, S.A. Offset frequency dynamics and phase noise properties of a self-referenced $10 \mathrm{GHz}$ Ti:sapphire frequency comb. Opt. Express 2011, 19, 18440-18451.

109. Peters, R.; Petermann, K.; Huber, G. A New Mixed Sesquioxide Yb:LuScO 3 : Spectroscopic Properties and Highly Efficient Thin-Disk Laser Operation. In Advanced Solid-State Photonics; OSA Technical Digest Series: Denver, CO, USA, 2009; paper MC4.

(C) 2013 by the authors; licensee MDPI, Basel, Switzerland. This article is an open access article distributed under the terms and conditions of the Creative Commons Attribution license (http://creativecommons.org/licenses/by/3.0/). 